\title{
Small-scale clumps in the galactic halo and dark matter annihilation
}

\author{
Veniamin Berezinsky ${ }^{\text {* }}$ \\ INFN, Laboratori Nazionali del Gran Sasso, I-67010 Assergi (AQ), Italy \\ and Institute for Nuclear Research of the RAS, Moscow, Russia
}

Vyacheslav Dokuchaev and Yury Eroshenkd

Institute for Nuclear Research of the RAS, Moscow, Russia

(Dated: November 6, 2018)

\begin{abstract}
Production of small-scale DM clumps is studied in the standard cosmological scenario with an inflation-produced primeval fluctuation spectrum. Special attention is given to the three following problems: (i) The mass spectrum of small-scale clumps with $M \lesssim 10^{3} M_{\odot}$ is calculated with tidal destruction of the clumps taken into account within the hierarchical model of clump structure. Only $0.1-0.5 \%$ of small clumps survive the stage of tidal destruction in each logarithmic mass interval $\Delta \ln M \sim 1$. (ii) The mass distribution of clumps has a cutoff at $M_{\min }$ due to diffusion of DM particles out of a fluctuation and free streaming at later stage. $M_{\min }$ is a model dependent quantity. In the case the neutralino, considered as a pure bino, is a DM particle, $M_{\min } \sim 10^{-8} M_{\odot}$. (iii) The evolution of density profile in a DM clump does not result in the singularity because of formation of the core under influence of tidal interaction. The radius of the core is $R_{c} \sim 0.1 R$, where $R$ is radius of the clump. The applications for annihilation of DM particles in the Galactic halo are studied. The number density of clumps as a function of their mass, radius and distance to the Galactic center is presented. The enhancement of annihilation signal due to clumpiness, valid for arbitrary DM particles, is calculated. In spite of small survival probability, the global annihilation signal in most cases is dominated by clumps. For observationally preferable value of index of primeval fluctuation spectrum $n_{p} \approx 1$, the enhancement of annihilation signal is described by factor $2-5$ for different density profiles in a clump.
\end{abstract}

PACS numbers: 12.60.Jv, 95.35.+d, 98.35.Gi

\section{INTRODUCTION}

Both analytic calculations [1, 2] and numerical simulations [3, 4, 5] predict existence of dark matter clumps in the Galactic halo. The density profile in these clumps according to analytic calculations [6, 7, 8, 9] and numerical simulations [4, 10] is $\rho(r) \propto r^{-\beta}$. An average density of the dark matter (DM) in a galactic halo itself also exhibits a similar density profile (relative to a galactic center) in the both approaches. The DM profiles in clusters of galaxies is discussed in 11] and in references therein. In the analytic approach of Gurevich and Zybin (see review [9] and references therein) the density profiles are predicted to be universal, with $\beta \approx 1.7-1.9$ for clumps, galaxies and two-point correlation functions of galaxies. In the numerical simulations the density profiles can be evaluated only for the relatively large scales due to the limited mass resolution. The value of $\beta$ differs in different simulations from $\beta=1.0$ 10] to $\beta=1.5$ [3] and may be non-universal for the objects of different mass scales [12]. An attempt of analytical explanation of the results of numerical simulations has been performed in 13, 14]. The phase-space density profiles of DM halos are investigated in [15].

\footnotetext{
*Electronic address: berezinsky@lngs.infn.it

${ }^{\dagger}$ Electronic address: dokuchaev@inr.npd.ac.ru

${ }^{\ddagger}$ Electronic address: erosh@inr.npd.ac.ru
}

A central cusp in the Galactic halo and the smaller scale clumps result in the enhancement of DM annihilation rate and thus in the stronger signals in the form of gamma-rays, radio emission, positrons and antiprotons. The gamma-ray and radio signal from central cusp in the Galactic halo has been first discussed in 16, 17]. Recently this problem was examined in [18, 19, 20, 21, 22]. The enhancement of DM annihilation rate due to the clumpiness of DM halo was first pointed out in [1]. Neutralino annihilation in clumps can result in the very large diffuse gamma-ray flux 23] in the model of the clumpy DM by Gurevich et al. 2]. Calculations of positron and antiproton production in the clumpy DM halo have been performed e. g. in [18] (see also [24, 25, 26]). Recently the annihilation of DM in the clumps has been studied in 27, 28, 29, 30, 31, 32. The synchrotron flux from DM annihilation products in clumps in the presence of the Galactic magnetic field is considered in [33]. Constraints on the DM clumpiness in the halos from heating of the disk galaxies is examined in [34, 35].

The main purpose of this work is evaluation of the enhancement of the annihilation signal due to presence of the small clumps of DM in the Galactic halo.

The small-scale self-gravitating Dark Matter Clumps, which will be referred to as DMCs or simply as clumps, may be formed in the early universe due to several mechanisms. These DMCs may be formed (i) by the growth of the adiabatic or isothermal fluctuations (originated at inflation) during the matter-dominated epoch; or (ii) from the density fluctuations in the models with topological 
defects (cosmic strings and domain walls) [1]; or (iii) during the radiation dominating era from the nonlinear isothermal fluctuations (originated by phase transitions in early Universe) 36] or from the large amplitude adiabatic fluctuations [37].

In this paper we shall consider only the most conservative case of adiabatic fluctuations which enter the nonlinear stage of evolution at the matter dominated epoch with the inflation-induced initial power-law power spectrum.

Small-scale clumps are formed only if the fluctuation amplitudes in the spectrum are large enough at the corresponding small scales. The inflation models predict the power-law primeval fluctuation spectrum. If the power-law index $n_{p} \geq 1$, DMCs are formed in a wide range of scales. During the universe expansion the small clumps are captured by the larger ones, and the larger clumps consist of the smaller ones and of continuously distributed DM. The convenient analytic formalism, which describes statistically this hierarchical clustering, is the Press-Schechter theory [38] and its extensions, in particular 'excursion set' formalism developed by Bond et al. 39] (for the clear introduction see [40]). However, this theory does not include the important process of the tidal destruction of small clumps inside the bigger ones. We take into account this process in Sec. $\nabla]$ and obtain the mass function for the small-scale DMCs in the Galactic halo. In the case of the power-law spectrum only a small fraction of the captured clumps survives, but even this small fraction is enough to dominate the total annihilation rate in the Galactic halo.

In the hierarchical theory of large-scale structure formation in the Universe the first formed objects have some minimal mass $M_{\min }$. The value of this mass is determined by the spectrum of initial fluctuations and by the properties of DM particles [2, 41]. This value is crucial for calculation of the DM annihilation rate. The estimates of $M_{\text {min }}$ existing in the literature for neutralino DM are substantially different, from $M_{\min } \sim 10^{-12} M_{\odot}$ in 42 to $M_{\min } \sim\left(10^{-7}-10^{-6}\right) M_{\odot}$ in $[43$. In Sec. III we present our calculations and discuss the previous results.

The DM annihilation rate crucially depends on the density profile $\rho(r) \propto r^{-\beta}$ of DM particles in a clump and on the distance $R_{c}$ where the density growth is cut off. This region is called the core. The radius of the core has been estimated in the literature in the different approximations. The estimation, $R_{c} / R \sim \delta_{\text {eq }}^{3}$, where $\delta_{\text {eq }}$ is the density fluctuation amplitude at the end of radiation dominated epoch, has been obtained in [9]. It was found from behavior of the damped mode of nonlinear fluctuations. A black hole or baryonic core in the center of the DMC can strongly affect the density saturation at $r \rightarrow 0$ for a very massive DMC 9, 19, 20, 21]. Calculations [16, 23] of the inward flux of DM particles into the dense central region of DMC also result, due to annihilation of DM particles, in a very small radius of the central core $R_{c}$. The above mentioned process is essential for the formation of the DMC core only in the case of almost perfectly spherically symmetrical clump.

We shall estimate the radius of the core imposed by tidal interaction, which gives the largest $R_{c}$ among those known in the literature. In the spherically symmetric self-gravitating clump on the stage of its formation the non-dissipative DM particles are moving nearly radially in the oscillation regime. The presence of a non-spherical (tidal) external gravitational field causes the deflection of particle trajectories in the clump from the radial ones. This process prevents the development of the central singularity in a clump and results in the core formation.

During the radiation dominated epoch the small fluctuations $\delta=\delta \rho / \rho \ll 1$ grow very slowly. At the matter dominated stage these fluctuations start to grow fast [4] in the regime $\delta \propto t^{2 / 3}$. Fluctuations get detached from the general cosmological expansion and start contracting after reaching the nonlinear value, $\delta \geq 1$. These nonlinear fluctuations form finally the DM clumps. The analytic studies of nonlinear evolution of fluctuations have been performed by many authors. One of the most detailed analytical approach was developed by Gurevich and Zybin [9]. In this formalism at the certain moment of gravitational contraction the density singularity forms in the center of a nonlinear fluctuation. From this singularity point the density caustics (i. e. the boundary of regions with a different number of streams) expand outward. The secondary caustics appear inside the primary ones and their number increases fast with time. This multi-stream instability has been discovered and studied in detail in [9]. It was demonstrated that the stationary universal density profile $\rho(r) \propto r^{-\beta}$ with $\beta=1.7-1.9$ is formed as a result of streams mixing. The maximum density of DM particles in a clump is reached at the center.

In our consideration of the clump formation we shall follow for convenience the theoretical scenario of Gurevich and Zybin [9]. However, the effects of tidal interaction, which is the main result of our work, are valid for a much broader class of scenarios.

The processes described above are valid for all DM particles which can be considered as non-dissipative. The signal production depends on the annihilation crosssection and thus on the nature of DM particles. However our strategy is to calculate the enhancement of the signal due to the halo clumpiness in comparison with an isotropic unclumped distribution of DM. As a guide we shall take neutralino as DM particle, but essentially our results for enhancement of the annihilation signal are relevant for a wide class of other DM particles.

We perform our calculations for cosmological model with the matter density $\Omega_{\mathrm{m}}=0.3$ and the cosmological term $\Omega_{\Lambda}=1-\Omega_{\mathrm{m}} \simeq 0.7$. The presence of $\Lambda$ term influences only the value of $\rho_{\text {eq }}$ and does not affect the formation of low mass DMCs. This is because the $\Lambda$ term contributes negligibly to the total cosmological density at time scales when the low-mass DMCs formation occurs. We shall use index 'eq' for the values at the moment of equality (i. e. transition from the radiation dominated 
to matter dominated epoch). We shall use the Hubble constant $70 \mathrm{~km} \mathrm{~s}^{-1} \mathrm{Mpc}^{-1}$.

\section{ENHANCEMENT OF ANNIHILATION SIGNAL DUE TO CLUMPS}

Let us consider a DM clump with the internal density profile $\rho_{\text {int }}(r)$ and a total mass $M=\int 4 \pi r^{2} \rho_{\text {int }}(r) d r$. An annihilation rate in a single clump is given by

$$
\dot{N}_{\mathrm{cl}}=4 \pi \int_{0}^{\infty} r^{2} d r \rho_{\mathrm{int}}^{2}(r) m_{\chi}^{-2}\left\langle\sigma_{\mathrm{ann}} v\right\rangle=\frac{3}{4 \pi} \frac{\left\langle\sigma_{\mathrm{ann}} v\right\rangle}{m_{\chi}^{2}} \frac{M^{2}}{R^{3}} S
$$

where $m_{\chi}$ is mass of a DM particle (being not necessarily neutralino), $v$ is relative velocity of two DM particles at the collision, $\sigma_{\text {ann }}$ is annihilation cross-section and $R$ is virial radius of a clump. Function $S$ is determined by Eq. (11) and depends on DM distribution in a clump, in particular $S=1$ for the simplest case of an uniform clump, when $\rho_{\text {int }}(r)=$ const at $r \leq R$ and $\rho_{\text {int }}(r)=0$ at $r>R$.

An expansion of $\left\langle\sigma_{\mathrm{ann}} v\right\rangle$ over the relative velocity $v$ of two DM particles has a form

$$
\left\langle\sigma_{\mathrm{ann}} v\right\rangle=a+b v^{2}+c v^{4}+\ldots
$$

where $a$ has a contribution of s-wave amplitude only, and $b$ - from both s- and p- waves. Since $v$ is very small, $\left\langle\sigma_{\mathrm{ann}} v\right\rangle$ can be put out of the integral in Eq. (1).

We shall use the following parametrization of the density profile in a clump

$$
\rho_{\text {int }}(r)=\left\{\begin{array}{lr}
\rho_{c}, & r<R_{c} ; \\
\rho_{c}\left(\frac{r}{R_{c}}\right)^{-\beta}, R_{c}<r<R ; \\
0, & r>R .
\end{array}\right.
$$

Using $\rho_{\text {int }}(r)$ from Eq. (3) it is easy to calculate $S$ from Eq. (11) as

$$
S\left(x_{c}, \beta\right)=\frac{(3-\beta)^{2}}{3(2 \beta-3)}\left(\frac{2 \beta}{3} x_{c}^{3-2 \beta}-1\right)\left(1-\frac{\beta}{3} x_{c}^{3-\beta}\right)^{-2},
$$

where $x_{c}=R_{c} / R$. Another approach to the parametrization of clump structure has been used in [29].

There is distribution of clumps in the Galactic halo at least over three parameters, mass $M$, radius $R$, and distance from the Galactic Center $l: n_{\mathrm{cl}}(M, R, l)$. This distribution can also depend on the parameters which describe the internal structure of the clumps, $\beta$ and $x_{c}$, from Eq. (3). We shall discuss this dependence in Sec.IV] In particular it will be demonstrated that $x_{c}=x_{c}(M, R)$, while $\beta$ is the universal constant. Thus the differential number density of DMCs in the halo can be written as:

$$
d N_{\mathrm{cl}}=n_{\mathrm{cl}}(l, M, R) d^{3} l d M d R,
$$

The observed signal at the position of the Earth from DM particle annihilation in the clumps is proportional to the quantity

$$
\begin{aligned}
I_{\mathrm{cl}}= & \frac{1}{4 \pi} \int_{0}^{\pi} d \zeta \sin \zeta \int_{0}^{r_{\max }(\zeta)} \frac{2 \pi r^{2} d r}{r^{2}} \int_{M_{\min }}^{M_{\max }} d M \int_{R_{\min }}^{R_{\max }} d R \\
& \times n_{\mathrm{cl}}(l(\zeta, r), M, R) \dot{N}_{\mathrm{cl}}(M, R),
\end{aligned}
$$

where $r$ is distance from the Sun (Earth) to a clump and $\zeta$ is angle between the line of observation and the direction to the Galactic center. The distance $l$ between a clump and the Galactic center can be given in terms of $r, r_{\odot}$ (distance from the Sun to the Galactic center) and $\zeta$ as $l(\zeta, r)=\left(r^{2}+r_{\odot}^{2}-2 r r_{\odot} \cos \zeta\right)^{1 / 2}$, and the maximum distance from the Sun to the outer halo border in the direction of $\zeta, r_{\max }(\zeta)=\left(R_{H}^{2}-r_{\odot}^{2} \sin ^{2} \zeta\right)^{1 / 2}$, where $R_{H} \sim 100 \mathrm{kpc}$ is the Galactic halo virial radius and $r_{\odot}=8.5 \mathrm{kpc}$ is the distance from the Sun to the Galactic center.

Additional annihilation signal is given by unclumpy DM in the halo with homogeneous (i.e. smoothly spread) density $\rho_{\mathrm{DM}}(l)$, where $l$ is a distance to the Galactic Center.

$$
I_{\mathrm{hom}}=\frac{\left\langle\sigma_{\mathrm{ann}} v\right\rangle}{2} \int_{0}^{\pi} d \zeta \sin \zeta \int_{0}^{r_{\max }(\zeta)} d r \rho_{\mathrm{DM}}^{2}(l(\zeta, r)) / m_{\chi}^{2} .
$$

The enhancement $\eta$ of the signal due to a presence of clumps is given by

$$
\eta=\frac{I_{\mathrm{cl}}+I_{\mathrm{hom}}}{I_{\mathrm{hom}}}
$$

This quantity describes the global enhancement of the annihilation signal observed at the Earth (e. g. the flux of radio, gamma, and neutrino radiations) as compared with usual calculations from annihilation of unclumpy DM. From Eqs. (8), (7) and (6) one can see that enhancement $\eta$ does not depend on the properties of DM particles, in particular on the annihilation cross-section, and is fully determined by the parameters of DM clumpiness. The further exact calculations in this paper will be performed using Eqs. (6)-(8), but now we shall turn to the approximate expression for $\eta$.

We shall accept now the simplifying assumptions. We assume that space density of clumps in the halo, $n_{\mathrm{cl}}(l)$ is proportional to the unclumpy DM density, $\rho_{\mathrm{DM}}(l)$ : $n_{\mathrm{cl}}(l)=\xi \rho_{\mathrm{DM}}(l) / M$ with $\xi \ll 1$. This assumption holds with a good accuracy for the small-scale clumps. In contrast, the distribution of large-scale clumps obtained in the numerical simulations [45] is rather different from the density distribution of the small clumps, especially in a central part of the halo because of the tidal disruption of clumps there. However, the clump signal is determined mostly by clumps of the minimal mass. We neglect here the distribution of clumps over $M$ and $R$. Instead we shall use a mean density of DM particles inside a clump 
$\bar{\rho}_{\text {int }}=3 M / 4 \pi R^{3}$. Finally, we shall introduce for convenience the effective density of DM particles in the halo defined as

$$
\tilde{\rho}_{\mathrm{DM}} \equiv \frac{\int_{0}^{\pi} d \zeta \sin \zeta \int_{0}^{r_{\max }(\zeta)} d r \rho_{\mathrm{DM}}^{2}(l(\zeta, r))}{\int_{0}^{\pi} d \zeta \sin \zeta \int_{0}^{r_{\max }(\zeta)} d r \rho_{\mathrm{DM}}(l(\zeta, r))} .
$$

As a result, we obtain for an enhancement factor the convenient estimate

$$
\eta \approx 1+\xi S\left(x_{c}, \beta\right) \frac{\bar{\rho}_{\text {int }}}{\tilde{\rho}_{\mathrm{DM}}},
$$

where $\xi$ is a fraction of DM in the form of clumps (see above) and $S\left(x_{c}, \beta\right)$ is given by Eq. (4). For typical parameters (see details in the following sections) $n_{p} \simeq 1$, $\beta \simeq 1.8, x_{c} \simeq 0.05, S\left(x_{c}, \beta\right) \simeq 5, \tilde{\rho}_{H} \sim \rho_{\mathrm{DM}}\left(r_{\odot}\right) \sim$ $0.3 \mathrm{GeV} \mathrm{cm}^{-3}, \bar{\rho}_{\text {int }} \sim 2 \times 10^{-22} \mathrm{~g} \mathrm{~cm}^{-3}, \xi \sim 0.001$, the numerical estimate $\eta \sim 3$ follows from Eq. (10).

\section{CLUMPS OF MINIMAL MASSES}

The number of clumps in the halo increases at small clump masses $M$, and the signal from clumps $I_{\mathrm{cl}}$ crucially depends on $M_{\min }$ in the clump distribution as Eq. (6) shows. The value of $M_{\min }$ is determined by a leakage of DM particles from the overdense fluctuations in the early universe. We shall describe first this process qualitatively and then present numerical calculations.

CDM particles at high temperature $T>T_{f} \sim 0.05 m_{\chi}$ are in the thermodynamical (chemical) equilibrium with cosmic plasma, when their number density is determined by temperature. After freezing at $t>t_{f}$ and $T<T_{f}$, the DM particles remain for some time in kinetic equilibrium with plasma, when the temperature of CDM particles $T_{\chi}$ is equal to temperature of plasma $T$, but number density $n_{\chi}$ is determined by freezing concentration and expansion of the universe. At this stage the CDM particles are not perfectly coupled to the cosmic plasma. Collisions between a CDM particle and fast particles of ambient plasma result in exchange of momenta and a CDM particle diffuses in the space. Due to diffusion the DM particles leak from the small-scale fluctuations and thus their distribution obtain a cutoff at the minimal mass $M_{D}$.

When the energy relaxation time for DM particles $\tau_{\text {rel }}$ becomes larger than the Hubble time $H^{-1}(t)$, the DM particles get out of the kinetic equilibrium. This conditions determines the time of kinetic decoupling $t_{d}$. At $t \geq t_{d}$ the CDM matter particles are moving in the free streaming regime and all fluctuations on the scale of

$$
\lambda_{\mathrm{fs}}=a\left(t_{0}\right) \int_{t_{d}}^{t_{0}} \frac{v\left(t^{\prime}\right) d t^{\prime}}{a\left(t^{\prime}\right)}
$$

and smaller are washed away [here $a(t)$ is the scaling factor of expanding universe and $v(t)$ is velocity of a DM particle at epoch $t$. The corresponding minimal mass at epoch $t_{0}$,

$$
M_{\mathrm{fs}}=\frac{4 \pi}{3} \rho_{\chi}\left(t_{0}\right) \lambda_{\mathrm{fs}}^{3}
$$

is much larger than $M_{D}$. Numerical calculations below (for neutralino) show that $M_{D}$ is close to $M_{\text {min }}$ from [42] and $M_{\mathrm{fs}}$ to $M_{\mathrm{min}}$ from [43].

The calculation of the minimal mass $M_{\min }$ in the mass spectrum of fluctuations is obviously model dependent. As the DM particle we shall consider the neutralino $\chi$, for which we take the pure bino state $(\chi=\tilde{B})$. As calculations below show the temperature of kinetic decoupling for a reasonable range of parameters is $T_{d} \sim 100 \mathrm{MeV}$, and thus we can consider cosmic plasma consisting of relativistic electrons, positrons, neutrinos and photons in thermal equilibrium.

The cross-sections for scattering of bino off left (right) electron and left neutrino are given in the Appendix A. A cross-section for $\nu \chi$ scattering is given by Eq. A5 and for $e \chi$ scattering it is 17 times larger, if to assume equal masses of selectrons and sneutrinos (we shall use $\tilde{m}$ for the both left and right selectron and sneutrino masses, and $\tilde{M}^{2}=\tilde{m}^{2}-m_{\chi}^{2}$ ).

First of all we shall calculate the moment $t_{d}$ and temperature $T_{d}$ of kinetic decoupling of neutralino, using condition

$$
\frac{1}{\tau_{\text {rel }}} \simeq H(t)
$$

where $H(t)=1 /(2 t)$ is the Hubble constant and $\tau_{\text {rel }}(T)$ is the energy relaxation time for neutralino at temperature of electron-neutrino gas $T$. The relaxation time $\tau_{\text {rel }}$ is determined by collisions of neutralino with fermions $\nu_{L}, e_{L}$ and $e_{R}$. Neutralino can be considered as particle at rest because the rest system coincides with the center-of-mass system with the accuracy of order $\sqrt{T / m_{\chi}}$. Let $\delta p$ is the neutralino momentum obtained in one scattering:

$$
(\delta p)^{2}=2 \omega^{2}(1-\cos \theta)
$$

where $\omega$ and $\theta$ is neutrino energy and scattering angle, respectively.

Let us introduce the number density of relativistic fermions with one polarization and with energy $\omega$ :

$$
n_{0}(\omega)=\frac{1}{2 \pi^{2}} \frac{\omega^{2}}{e^{\omega / T}+1},
$$

Then for the energy relaxation time $\tau_{\text {rel }}$ we have

$$
\frac{1}{\tau_{\mathrm{rel}}}=\frac{1}{E_{k}} \frac{d E_{k}}{d t}=\frac{40}{2 E_{k} m_{\chi}} \int d \Omega \int d \omega n_{0}(\omega)\left(\frac{d \sigma_{\mathrm{el}}}{d \Omega}\right)_{f_{L} \chi}(\delta p)^{2},
$$

where $E_{k} \simeq(3 / 2) T$ is a mean kinetic energy of neutralino, and $\left(d \sigma_{\mathrm{el}} / d \Omega\right)_{f_{L} \chi}$ is given by Eq. (A5). The 
number 40 in Eq. (16) is obtained by counting of degrees of freedom: three neutrinos and antineutrinos (or $\nu_{L}^{c}$ in case of Majorana neutrinos) give $6, e_{L}$ and $e_{L}^{c}$ give 2 and two right (singlet) states for electron and positron gives 34, because their cross-sections are 17 times larger.

After integration in Eq. (16) we obtain

$$
\frac{1}{\tau_{\text {rel }}}=\frac{40 \Gamma(7) \alpha_{\mathrm{e} . \mathrm{m} .}^{2}}{9 \pi \cos ^{4} \theta_{\mathrm{W}}} \frac{T^{6}}{\tilde{M}^{4} m_{\chi}}
$$

Using Eq. (13) and connection between age and temperature of the universe

$$
t=\frac{2.42}{\sqrt{g_{*}}}\left(\frac{T}{1 \mathrm{MeV}}\right)^{-2} \mathrm{~s}
$$

where $g_{*}$ is number of degrees of freedom, we obtain numerically:

$$
t_{d}=3.5 \times 10^{-5}\left(\frac{m_{\chi}}{100 \mathrm{GeV}}\right)^{-1 / 2}\left(\frac{\tilde{M}}{1 \mathrm{TeV}}\right)^{-2}\left(\frac{g_{*}}{10}\right)^{-3 / 4} \mathrm{~s}
$$

and

$$
T_{d}=150\left(\frac{m_{\chi}}{100 \mathrm{GeV}}\right)^{1 / 4}\left(\frac{\tilde{M}}{1 \mathrm{TeV}}\right)\left(\frac{g_{*}}{10}\right)^{1 / 8} \mathrm{MeV}
$$

We shall present in this section the calculations made in physically transparent way, considering the diffusion leaking of neutralinos at the stage of kinetic equilibrium and free streaming when neutralinos get out of kinetic equilibrium. In Appendix B we shall study both stages together in the formalism of kinetic equation, as it has been done in [42]. Though our methods are not identical, their comparison implies that the absence of free streaming is responsible for the contradiction with different $M_{\min }$ discussed above. The independent approach in Appendix B confirms the results obtained below.

\section{A. Diffusion cutoff of the mass spectrum}

We can come over now to the calculation of $M_{D}$, the minimal mass in the fluctuation spectrum caused by diffusion of neutralinos out of an overdense fluctuation. We calculate the diffusion coefficient using the method given in [46] (\$12). Consider a neutralino moving with a nonrelativistic velocity $\vec{v}$. In the rest system of this particle the momentum distribution of relativistic fermions is anisotropic:

$$
n(\vec{p}) d^{3} p=\frac{d \Omega_{\alpha} p^{2} d p}{(2 \pi)^{3}} \frac{1}{e^{p(1+v \cos \alpha) / T}+1},
$$

where $\alpha$ is the angle between the directions of $\vec{v}$ and momentum of incoming fermion.

The momentum transfer in a single scattering equals to $\vec{p}(1-\cos \theta)$ after averaging over the azimuthal angles.
A corresponding friction force experienced by the neutralino is

$$
\begin{aligned}
\vec{f}_{r} & =40 \int d \Omega_{\theta} \int d^{3} p n(\vec{p})\left(\frac{d \sigma_{\mathrm{el}}}{d \Omega_{\theta}}\right)_{f_{l} \chi} \vec{p}(1-\cos \theta) \\
& =-B^{-1} \vec{v}
\end{aligned}
$$

where $B$ is a particle mobility and factor 40 takes into account scattering on all fermions as in Eq. (17). Then the diffusion coefficient is

$$
D=B T=\frac{3 \pi \cos ^{4} \theta_{\mathrm{W}} \tilde{M}^{4}}{40 \Gamma(6) \alpha_{\mathrm{e} . \mathrm{m} .}^{2} T^{5}}
$$

Diffusion equation in the comoving system has a form

$$
\frac{\partial \delta(\vec{x}, t)}{\partial t}=\frac{D(t)}{a^{2}(t)} \Delta_{\vec{x}} \delta(\vec{x}, t)
$$

Diffusion coefficient $D(t)$ is time-dependent because of $T(t)$. Solution of Eq. (24) for the Fourier component is

$$
\delta_{\vec{k}}(t)=\delta_{\vec{k}}\left(t_{f}\right) \exp \left\{-k^{2} C g_{*}^{5 / 4} \tilde{M}^{4}\left(t^{5 / 2}-t_{f}^{5 / 2}\right)\right\},
$$

where $C=$ const. The factor $C g_{*}^{5 / 4} \tilde{M}^{4}\left(t^{5 / 2}-t_{f}^{5 / 2}\right)$ in front of $k^{2}$ in Eq. (25) is the diffusion length squared $\lambda_{\mathrm{D}}^{2}(t) / a^{2}(t)$ in the comoving coordinates. This value determines the minimal mass in the density perturbation spectrum due to diffusion of neutralinos from a fluctuation:

$$
\begin{aligned}
M_{\mathrm{D}} & =\frac{4 \pi}{3} \rho_{\chi}\left(t_{d}\right) \lambda_{\mathrm{D}}^{3}\left(t_{d}\right)=4.3 \times 10^{-13}\left(\frac{m_{\chi}}{100 \mathrm{GeV}}\right)^{-15 / 8} \\
& \times\left(\frac{\tilde{M}}{1 \mathrm{TeV}}\right)^{-3 / 2}\left(\frac{g_{*}}{10}\right)^{-15 / 16} M_{\odot} .
\end{aligned}
$$

The functional dependence of Eq. (25) and numerical value of (26) obtained in diffusion approximation coincide with the corresponding results obtained by different method in 42].

\section{B. Free streaming cutoff of the mass spectrum}

We shall consider now the free streaming cutoff of the mass spectrum qualitatively described in the beginning of this section. We have given there an estimate of the minimal mass due to free streaming. In the accurate calculations below we shall take into account the angular and velocity distribution of leaking neutralinos, and exact dependence of $a(t)$ at age $\sim t_{\text {eq }}$, which affect the value of $M_{\mathrm{fs}}$.

After the moment of kinetic decoupling $t_{d}$, neutralinos move freely in the expanding universe background, $a(t) d \vec{x}=\vec{v}(t) d t$, where $\vec{x}$ is comoving particle coordinates. Coordinates $\vec{x}$ at some moment $t$ are determined 
by initial coordinates $\vec{q}$ and velocity $\vec{v}_{d}$ at the moment of kinetic decoupling $t_{d}$ :

$$
\vec{x}=\vec{f}\left(\vec{q}, \vec{v}_{d}, t\right)=\vec{q}+\int_{t_{d}}^{t} \frac{\vec{v}\left(t^{\prime}\right) d t^{\prime}}{a\left(t^{\prime}\right)}=\vec{q}+g(t) \vec{v}_{d},
$$

where

$$
g(t)=a\left(t_{d}\right) \int_{t_{d}}^{t} \frac{d t^{\prime}}{a^{2}\left(t^{\prime}\right)}
$$

$\vec{v}(t)=\vec{v}_{d} a\left(t_{d}\right) / a(t)$ for nonrelativistic particle. Now for the neutralino number density at the point $\vec{x}$ we have

$$
\begin{aligned}
n(\vec{x}, t) & =\int d^{3} v_{d} \phi\left(\vec{v}_{d}\right) \sum_{\vec{q}_{*}} n\left(\vec{q}_{*}, t_{d}\right)\left|\frac{D \vec{f}}{D \vec{q}}\right|_{\vec{q}=\vec{q}_{*}} \\
& =\int d^{3} v_{d} \phi\left(\vec{v}_{d}\right) \int d^{3} q n\left(\vec{q}, t_{d}\right) \delta^{(3)}\left(\vec{x}-\vec{f}\left(\vec{q}, \vec{v}_{d}, t\right)\right),
\end{aligned}
$$

where $\delta^{(3)}$ is the Dirac delta-function, $D \vec{f} / D \vec{q}$ is the Jacobian and $\phi\left(\vec{v}_{d}\right)$ is neutralino velocity distribution function at the moment $t_{d}$. Summation in (29) goes over all roots $\vec{q}_{*}$ of the equation $\vec{x}=\vec{f}\left(\vec{q}, \vec{v}_{d}, t\right)$ from (27). This sum in fact has only one term because the function $f\left(\vec{q}, \vec{v}_{d}, t\right)$ in (27) is a single-valued one.

From (27) and (29) we find the Fourier component

$$
n_{\vec{k}}(t)=n_{\vec{k}}\left(t_{d}\right) \int d^{3} v_{d} \phi\left(\vec{v}_{d}\right) e^{-i \vec{k} \vec{v}_{d} g(t)} .
$$

Assuming velocity distribution at the moment of decoupling $\phi\left(\vec{v}_{d}\right)$ to be Maxwellian

$$
\phi\left(\vec{v}_{d}\right)=\left(\frac{m_{\chi}}{2 \pi T_{d}}\right)^{3 / 2} \exp \left\{-\frac{m_{\chi} v_{d}^{2}}{2 T_{d}}\right\},
$$

we obtain

$$
n_{\vec{k}}(t)=n_{\vec{k}}\left(t_{d}\right) e^{-\frac{1}{2} k^{2} g^{2}(t) \frac{T_{d}}{m_{\chi}}},
$$

i.e. up to the moment $t$ all perturbations are washed out by free streaming inside the physical length-scale

$$
\lambda_{\mathrm{fs}}(t)=a(t) g(t)\left(\frac{T_{d}}{m_{\chi}}\right)^{1 / 2} .
$$

This length-scale corresponds to the clump of the minimal mass

$$
M_{\mathrm{fs}}(t)=\frac{4 \pi}{3} \rho_{\chi}(t) \lambda_{\mathrm{fs}}^{3}(t)
$$

where $\rho_{\chi}(t)=\rho_{\mathrm{eq}} a_{\mathrm{eq}}^{3} / a^{3}(t)$. The time dependence of $M_{\mathrm{fs}}(t)$ is regulated by $a(t)$. At the radiation dominated epoch, $M_{\mathrm{fs}}(t)$ grows logarithmically with time. This growth is saturated at the matter dominated epoch. The resulting $M_{\min }$ at $t_{0}$ can be easily calculated using $a(t)$ as solution of the Friedman equation:

$$
\begin{aligned}
& a(\eta)=a_{\mathrm{eq}}\left[2 \frac{\eta}{\eta_{*}}+\left(\frac{\eta}{\eta_{*}}\right)^{2}\right], \\
& t=a_{\mathrm{eq}} \eta_{*}\left[\left(\frac{\eta}{\eta_{*}}\right)^{2}+\frac{1}{3}\left(\frac{\eta}{\eta_{*}}\right)^{3}\right] .
\end{aligned}
$$

In these equations $\eta_{*}^{-2}=2 \pi G \rho_{\text {eq }} a_{\text {eq }}^{2} / 3, a_{\text {eq }}$ is the value of scaling factor at the moment $t_{\mathrm{eq}}$,

$$
\rho_{\text {eq }}=\rho_{0}\left(1+z_{\text {eq }}\right)^{3}=1.1 \times 10^{-19}\left(\frac{h}{0.7}\right)^{8}\left(\frac{\Omega_{m}}{0.3}\right)^{4} \mathrm{~g} \mathrm{~cm}^{-3} \text {, }
$$

$1+z_{\mathrm{eq}}=2.35 \times 10^{4} \Omega_{m} h^{2}$ and $\rho_{0}=1.9 \times$ $10^{-29} \Omega_{m} h^{2} \mathrm{~g} \mathrm{~cm}^{-3}$. The presence of cosmological constant $\Lambda$ affects only the value $\rho_{\text {eq }}$ and does not influence the evolution $M_{\mathrm{fs}}(t)$ because the contribution of $\Lambda$ to the total cosmological density is negligible at small $t$. Putting (35) into (28), we find after integration

$$
M_{\min }=\frac{\pi^{1 / 4}}{2^{19 / 4} 3^{1 / 4}} \frac{\rho_{\mathrm{eq}}^{1 / 4} t_{d}^{3 / 2}}{G^{3 / 4}}\left(\frac{T_{d}}{m_{\chi}}\right)^{3 / 2} \ln ^{3}\left\{\frac{24}{\pi G \rho_{\mathrm{eq}} t_{d}^{2}}\right\} .
$$

Using Eqs. (19) and (20) we obtain numerically

$$
\begin{aligned}
M_{\min }= & 1.5 \times 10^{-8}\left(\frac{m_{\chi}}{100 \mathrm{GeV}}\right)^{-15 / 8}\left(\frac{\tilde{M}}{1 \mathrm{TeV}}\right)^{-3 / 2} \\
& \times\left(\frac{g_{*}}{10}\right)^{-15 / 16}\left(\frac{\Lambda^{*}}{83}\right)^{3} M_{\odot}
\end{aligned}
$$

where $\Lambda^{*}$ is the logarithm from Eq. (37).

Our calculations agree well with [43] as far as the most important quantity $T_{d}$ is concerned (the scattering crosssection is involved only there). The calculation of $M_{\min }$ from $T_{d}$ in our case involves non-radial propagation of neutralinos in a fluctuation and their distribution over velocities, Eqs. (27) - (32). We include also the accurate time dependence of the scaling factor $a(t)$. The calculations in 43] follow the semi-quantitative scheme described in the beginning of Sec. III] At this stage of calculations we have a difference described by factor 7 .

In conclusion, in this section we have considered two processes of washing out the cosmological density perturbations in neutralino gas. The first process is the neutralino diffusion due to scattering off neutrinos, electrons and positrons. This process is effective until neutralinos are in the kinetic equilibrium with the cosmological plasma. Up to the moment of decoupling $t_{d}$ all perturbations with mass $M<M_{\mathrm{D}} \simeq 10^{-13}-10^{-12} M_{\odot}$ are washed out. The second process is neutralino free steaming. Starting later, at $t>t_{d}$, it washes out the larger perturbations with $M \leq M_{\mathrm{fs}}$ and determines $M_{\mathrm{min}}$ in the clump mass distribution at present epoch, as given by Eq. (38). 


\section{CORE OF A DARK MATTER CLUMP}

In this section we shall consider smearing of the singular density profile in a clump due to tidal forces and calculate the radius $R_{c}$ of the produced core.

Clumps, as well as galaxies, are originated near the maxima in cosmological density perturbations $\delta(\vec{r})=$ $(\rho(\vec{r})-\bar{\rho}) / \bar{\rho}$. At the matter dominated stage the density perturbations grow as $\delta \propto t^{2 / 3}$. In the nonlinear regime, $\delta \gtrsim 1$, the multi-flux instability develops in a clump and singular density profile is formed 9]. If velocity field in the central part of the clump is disturbed and becomes weakly nonradial, the flow is overturned, singularity does not form and density profile is smoothed. In [9] the core radius is estimated as $x_{c} \simeq \delta_{\mathrm{eq}}^{3} \ll 1$ from consideration of the perturbation of the velocity field due to damped mode of the cosmological density perturbations. Here $\delta_{\text {eq }}$ is an initial density fluctuation value at the end of radiation dominated epoch. In 23 the core is produced for spherically symmetric clump by inverse flow caused by annihilation of DM particles. We shall show here that these phenomena are not the main effects and that much stronger disturbance of the velocity field in the core is produced by tidal forces. These forces originate due to non-sphericity of the considered perturbation and presence of other fluctuations nearby, including a fluctuation of larger scale in which the considered fluctuation can be submerged.

The core formation in a fluctuation begins at the linear stage of evolution and continues at the beginning of non-linear stage. The tidal forces diminishes with time $t$ (see Eq. (53) below), while duration of this phase is proportional to $t$. Once the core is produced it is not destroyed in the evolution followed. The stage of the core formation continues approximately from $t_{\mathrm{e} q}$ to the time of maximal expansion $t_{s}$ and a little above, when a clump is detached from expansion of universe and evolves in the non-linear regime. Soon after this period, a clump enters the hierarchical stage of evolution, when the tidal forces can destroy it, but surviving clumps retain their cores.

Let us expand the gravitational potential in the series near the maximum of the density fluctuation taken as $\vec{r}=0$ at arbitrary time $t$ during the linear growth of density perturbations:

$$
\phi(\vec{r}, t)=\phi_{0}+\left.\frac{\partial \phi}{\partial r^{i}}\right|_{0} r^{i}+\left.\frac{1}{6} \Phi_{l l}\right|_{0} \delta_{i j} r^{i} r^{j}+\left.\frac{1}{2} T_{i j}\right|_{0} r^{i} r^{j}+\ldots,
$$

where

$$
\Phi_{i j}=\frac{\partial^{2} \phi(\vec{r})}{\partial r^{i} \partial r^{j}}, \quad T_{i j}=\Phi_{i j}-\frac{1}{3} \Phi_{l l} \delta_{i j}
$$

The first term of series in Eq. (39) does not influence the particle motion. The second term is zero as a condition of maximum density. The third term describes the spherically symmetric part of the potential (including the potential of the homogeneous background) and also the perturbation potential. It governs the radial motion of the particles. According to the Poisson equation one has

$$
\Phi_{l l}=\Delta \phi(\vec{r})=4 \pi G \bar{\rho}(1+\delta(\vec{r})) .
$$

Finally, the fourth term, which contains the traceless matrix $T_{i j}$, describes the tidal forces. They disturb the radial motion of the particles and result in production of the core.

We shall start with definitions and notation. We assume that density perturbations $\delta(\vec{r})$ are Gaussian with a power spectrum $P(k)$ :

$$
\left\langle\delta_{\vec{k}}^{*} \delta_{\vec{k}^{\prime}}\right\rangle=(2 \pi)^{3} P(k) \delta_{\mathrm{D}}^{(3)}\left(\vec{k}-\vec{k}^{\prime}\right), \quad \delta_{\vec{k}}=\int \delta(\vec{r}) e^{i \vec{k} \vec{r}} d^{3} r,
$$

where $\delta_{\mathrm{D}}^{(3)}\left(\vec{k}-\vec{k}^{\prime}\right)$ is the Dirac delta-function and angle brackets corresponds to ensemble averaging. The power spectrum $P_{\text {eq }}(k)$ at the time $t_{\text {eq }}$ is connected with the primordial power spectrum $P_{p}(k)$ (at the epochs before the horizon crossing) by relation $P_{\text {eq }}(k)=P_{p}(k) T^{2}(k)$, where $T(k)$ is the transfer function for cold dark matter (see e. g. [47]).

From Eq. (41) it follows that the power spectrum $P_{\Phi}(k)$ of potential perturbations is related to $P(k)$ as

$$
P_{\Phi}(k)=(4 \pi)^{2} G^{2} \bar{\rho}^{2} k^{-4} P(k) .
$$

Let us introduce the moments of spectrum $P(k)$

$$
\sigma_{(j)}^{2}=\frac{1}{2 \pi^{2}} \int_{0}^{\infty} k^{2} d k P(k) k^{2 j}
$$

and the similar moments $s_{(j)}^{2}$ for the perturbation field of the gravitational potential. Calculating the divergent moments for given mass $M$ we assume smoothing procedure of [47].

From Eq. (43) it follows that

$$
s_{(j)}^{2}=(4 \pi)^{2} G^{2} \bar{\rho}^{2} \sigma_{(j-2)}^{2}
$$

for $j \geq 2$. Let us define $\zeta_{i j}=\partial^{2} \delta(\vec{r}) / \partial r^{i} \partial r^{j}$. Then according to [4]], its mean value over the ensemble is

$$
\left\langle\zeta_{i j} \zeta_{k l}\right\rangle=\frac{\sigma_{(2)}^{2}}{15}\left(\delta_{i j} \delta_{k l}+\delta_{i k} \delta_{j l}+\delta_{i l} \delta_{j k}\right)
$$

which results in

$$
\left\langle T_{i j} T_{j i}\right\rangle=\frac{2}{3} s_{(2)}^{2}=\frac{2}{3}(4 \pi)^{2} G^{2} \bar{\rho}^{2} \sigma_{(0)}^{2},
$$

(in the following we shall use a notation $\left.\sigma \equiv \sigma_{(0)}\right)$. Let us introduce the important physical quantity $\nu$, the height of the peak density in units of dispersion (the peak-height):

$$
\nu=\delta_{\text {eq }} / \sigma_{\text {eq }}(M),
$$

where $\sigma_{\text {eq }}(M) \equiv \sigma\left(t_{\text {eq }}, M\right)$.

After introduction of these quantities we shall come over to calculation of DM particle velocities and core radius. The velocity $\vec{v}(t)$ is given by sum of radial velocity 
$\vec{v}_{\text {rad }}$ and an additional velocity $\vec{v}_{\text {tid }}$, which will be considered as a small correction caused by tidal forces. The radial velocity will be calculated without tidal interaction taking into account from the equation

$$
\frac{d \vec{v}_{\mathrm{rad}}}{d t}=-\operatorname{grad} \phi(r)
$$

where spherically symmetric potential $\phi(r)$ is given by the third term in r.h.s of Eq. (39). This equation determines the radial motion of the particle, and its solution is given in the parametric form as [44]:

$$
r=r_{s} \cos ^{2} \theta, \quad \theta+\frac{1}{2} \sin 2 \theta=\frac{2}{3}\left(\frac{5 \delta_{\mathrm{eq}}}{3}\right)^{3 / 2} \frac{t-t_{s}}{t_{\mathrm{eq}}} .
$$

The moment of maximum clump expansion $t_{s}$ and the distance $r=r_{s}$ at this moment are

$$
\frac{t_{s}}{t_{\mathrm{eq}}}=\frac{3 \pi}{4}\left(\frac{5 \delta_{\mathrm{eq}}}{3}\right)^{-3 / 2}, \quad \frac{r_{s}}{r_{\mathrm{eq}}}=\frac{3}{5 \delta_{\mathrm{eq}}},
$$

where $\delta_{\text {eq }}$ is the initial fluctuation value (at $t=t_{\text {eq }}$ ).

Tidal forces give rise to the additional velocity $\vec{v}_{\text {tid }}$. Its evolution is described by equation

$$
\frac{d v_{\mathrm{tid}, \mathrm{i}}}{d t}=-T_{i j}(t) r^{j}
$$

where in the linear approximation

$$
T_{i j}(t)=T_{i j}\left(t_{\mathrm{eq}}\right)\left(\frac{t}{t_{\mathrm{eq}}}\right)^{-4 / 3} .
$$

The linear approximation for tidal forces is justified because they are generated by the large-scale perturbations which become nonlinear later than the small-scale perturbation under consideration.

Now we find the value $\vec{v}_{\text {tid }}$ at the moment when the density nonlinearity sets in, $\delta \simeq 1$, or more exactly at the moment of a maximum expansion $t_{s}$. After integration of (52) with the help of Eq. (50) we obtain

$$
v_{\mathrm{tid}, \mathrm{i}}\left(t_{s}\right)=18^{1 / 3}\left(\frac{5 \delta_{\mathrm{eq}}}{3}\right)^{1 / 2} t_{\mathrm{eq}} f\left(\delta_{\mathrm{eq}}\right) T_{i j}\left(t_{\mathrm{eq}}\right) r^{j}\left(t_{s}\right),
$$

where the function

$$
f\left(\delta_{\mathrm{eq}}\right)=\frac{2}{3} \int_{\left(5 \delta_{\mathrm{eq}} / 3\right)^{1 / 2}}^{\pi / 2} d \phi\left(\phi-\frac{1}{2} \sin 2 \phi\right)^{-4 / 3} \sin ^{4} \phi
$$

is plotted in the Fig. 1] We may use approximately $f\left(\delta_{\mathrm{eq}}\right) \simeq 1$ for the values of $\delta_{\mathrm{eq}}$ at interest (asymptotically $f\left(\delta_{\text {eq }}\right) \rightarrow 1.32$ at $\left.\delta_{\text {eq }} \rightarrow 0\right)$.

To find the core radius $R_{c}$ of the clump we shall use the method similar to that in [9]. Since rot $\vec{v}_{\text {tid }}=0$ and $\operatorname{div} \vec{v}_{\text {tid }}=0$, the tensor $T_{i j}$ has the following diagonal form in the coordinate system connected with the main axes.

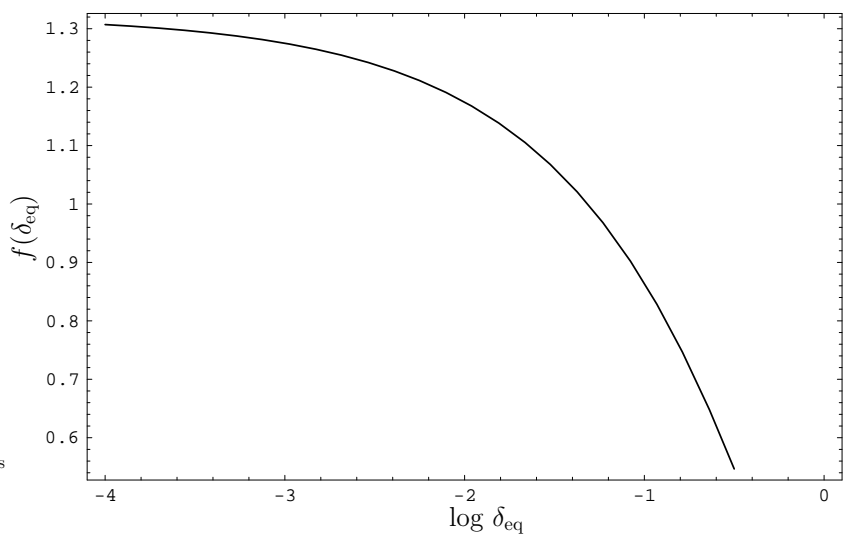

FIG. 1: Function $f\left(\delta_{\text {eq }}\right)$ defined by Eq. (55). The ratio of the core radius to the clump radius in typical case $\simeq 0.3 \nu^{-2} f\left(\delta_{\text {eq }}\right)$.

$$
T_{i j}^{\prime}=\left(\begin{array}{ccc}
\tau & & \\
& \tau & \\
& & -2 \tau
\end{array}\right)
$$

The value $\tau$ from Eq. (56) is connected with the core radius $R_{c}$ due to energy relation $\Delta E \simeq \Delta V$, where $\Delta E \simeq$ $\int d^{3} r \rho_{\text {int }}\left(t_{s}\right) v_{\text {tid }}^{2}\left(t_{s}\right) / 2$ is the work performed by tidal forces, and $\Delta V \simeq G M M_{c} / R$, where $M_{c} \sim 4 \pi R_{c}^{3} \rho_{c} / 3$, is potential energy of the core. It gives for the relative core radius

$$
x_{c} \simeq \frac{2^{2 / 3} 3^{10 / 3}}{\pi} \frac{\tau^{2} t_{\mathrm{eq}}^{2} \delta_{\mathrm{eq}}}{G \bar{\rho}_{\mathrm{int}}} f^{2}\left(\delta_{\mathrm{eq}}\right) .
$$

From invariance of the matrix trace relative to the change of coordinates one has

$$
\tau^{2}=T_{i j}^{\prime} T_{j i}^{\prime} / 6=T_{i j} T_{j i} / 6 .
$$

Since the correlator

$$
\left\langle T_{i j} \delta\right\rangle=\left\langle\left(\Phi_{i j}-\frac{1}{3} \Phi_{l l} \delta_{i j}\right)\left(\Phi_{l l}(4 \pi G \bar{\rho})^{-1}-1\right)\right\rangle=0,
$$

the quantities $\delta$ and $T_{i j}$ are statistically independent and we may average them independently. Averaging $\left\langle\tau^{2}\right\rangle$ over the tidal force field $T_{i j}$ with help of Eqs. (47) and (58), we obtain the main result of this section for the relative core radius:

$$
\begin{aligned}
x_{c}=\frac{R_{c}}{R} & \simeq \frac{\pi 2^{5 / 3} 3^{13 / 3}}{5^{3}} G \rho_{\mathrm{eq}} t_{\mathrm{eq}}^{2} \nu^{-2} f^{2}\left(\delta_{\mathrm{eq}}\right) \\
& \simeq 0.3 \nu^{-2} f^{2}\left(\delta_{\mathrm{eq}}\right),
\end{aligned}
$$

where $\nu$ is given by Eq. (48).

The fluctuations with $\nu \sim 0.5-0.6$ have $x_{c} \sim 1$, i.e. these fluctuations are practically destroyed by tidal interactions. Most of galactic clumps are formed from $\nu \sim 1$ peaks. As it will be demonstrated in Sec. VI those clumps that survive till now are characterized by $\nu \simeq 1.6$, but the main contribution to the annihilation 
signal is given by the clumps with $\nu \simeq 2.5$ for which $x_{c} \simeq 0.05$.

In an alternative approach for calculation of the core radius one may define $R_{c}$ as the minimum deviation of a typical particle trajectory from a center of the clump. After clump virialization a particle at the average distance $R / 2$ from the center has an angular momentum $m v_{\text {tid }} R / 2$. At $t>t_{s}$ the tidal force is already small and angular momentum is approximately concerved. Then at the minimum distance from the core, $R_{c}$, one has $R_{c} V \sim v_{\text {tid }} R / 2$, where $V=(2 G M / R)^{1 / 2}$ is the velocity of typical particle in the center of the virialized clump. Calculating $v_{\text {tid }}$ from Eq. (54) and using Eq. (47) one obtains

$$
x_{c} \simeq 0.15 \nu^{-1} f\left(\delta_{\mathrm{eq}}\right),
$$

which numerically is very close to Eq. (60) for typical values of $\nu \simeq 1-3$.

The core radius, given by the Eq. (60), is much larger than $x_{c} \simeq \delta_{\text {eq }}^{3}$ obtained in [9] and $x_{c}$ from [23]. The core radius found in 23] is valid only in a perfectly symmetric case.

Several remarks are in order.

Tidal forces prevent the appearance of singularity during evolution of the clump, but if such singularity has somehow appeared, tidal interactions cannot destroy it.

In the calculations above we operated with average tidal force, described by Eq. (47). In reality this force fluctuates due to positions of surrounding fluctuations which can overlap with the considered one or to be far away from it. As a result some clumps can be destroyed and those survived have different core radii $R_{c}$. This effect increases the annihilation signal.

We assume that DM distribution within the core is much flatter than $r^{-\beta}$. Between $R_{c}$ and the beginning of the asymptotic regime $\rho_{\text {int }} \propto r^{-\beta}$ there is a transition zone. During the hierarchical evolution (see next section) this zone expands due to tidal interaction in the hierarchical structures. However, this interaction cannot destroy the existing core.

The above calculations are also valid for the formation of galaxies. It is useful to compare Eq. (60) with observations of galaxies. In 47] the number of peaks in the Gaussian random field is confronted with the observed density of spiral and elliptical galaxies. It was found that these galaxies are formed mostly from peaks with $\nu \simeq 3$. According to Eq. (60) for these peaks $x_{c} \simeq 0.033$. The rotation curves in the central part of dwarf and Low Surface Brightness galaxies are measured [48, 49] and constant-density cores were revealed. Existing observations do not contradict the presence of extended uniform core with radius $R_{c}=x_{c} R \sim 3.3 \mathrm{kpc}$ in spiral and elliptical galaxies. However, at these distances the baryonic matter dominates, which makes the observation of DM core even more difficult. The extreme value $R_{c}=R$ corresponds to $\nu_{\min } \approx 0.55 f$. The Gaussian peaks with these $\nu$ are completely washed out by tidal forces and do not produce the gravitationally bound objects. The intermediate case $\nu \simeq 1$ corresponds to most numerous dwarf and irregular galaxies with the pronounced overdensity in the central core.

\section{TIDAL DESTRUCTION OF CLUMPS IN THE HIERARCHICAL MODEL}

In this section we shall study the destruction of clumps by the tidal interaction which occurs at the formation of the hierarchical structures, but long time before the galaxy formation. This interaction arises when two clumps pass near each other and when a clump moves in the external gravitational field of the bigger object (host) to which this clump belongs. In both cases a clump is exited by the external gravitational field, i. e. its constituent particles obtain additional velocities in the c. m. system. The clump is destroyed if its internal energy increase $\Delta E$ exceeds the corresponding total energy $|E| \sim G M^{2} / 2 R$. In Sec. $\nabla \mathrm{B} \mid$ we shall calculate the rate of excitation energy production due to both aforementioned processes. Respectively in Sec. $\nabla \mathbf{V}$ we shall calculate the survival probability for a clump in the hierarchical model, in which the smaller clumps are embedded in the bigger one, and the latter enters into a more bigger clump etc. But first we shall describe the necessary generalities and definitions.

\section{A. Generalities and definitions}

The formation of DM objects with a fixed mass $M$ at the linear stage is distributed over formation epochs $t_{f}$. In the spherical model of the Press-Schechter theory [38, 40] the density perturbation at the epoch of object formation is equal to $\delta_{c}=3(12 \pi)^{2 / 3} / 20 \simeq 1.686$ :

$$
\delta\left(M, t_{f}\right)=\delta_{c}
$$

Eq. (62) gives the formation criterion for DM objects. The formation criterion alone does not determine $t_{f}$ for a given mass $M$, because $\delta(M)$ has the Gaussian distribution. The formation time $t_{f}$ can be fixed by an additional condition, e. g. $\nu=1$ (see Eq. (48)). The DM objects which satisfy the formation criterion (62) and $\nu=1$, or equivalently $\sigma\left(M, t_{f}\right)=\delta_{c}$, will be referred to as the typical objects. For a given mass $M$ they are characterized by a fixed epoch of formation $t_{f}$. In some parts of our consideration we shall simplify the problem, assuming that clumps are typical instead of taking into account their distribution over $t_{f}$.

We confine ourselves here only to the power-law spectrum of fluctuations $P_{\mathrm{eq}}(k) \propto k^{n}$, in which case

$$
\sigma_{\text {eq }}(M) \propto M^{-(n+3) / 6} .
$$

The effective power-law index $n$ obtained from the expression above can be given as

$$
n=-3-6 \frac{\partial \ln \sigma_{\mathrm{eq}}(M)}{\partial \ln M} .
$$


In the case of an arbitrary $P(k)$ spectrum, the $\sigma_{\text {eq }}(M)$ has also an arbitrary dependence on $M$. Eq. (64) defines $n_{\text {eff }}$ for a some mass interval $\Delta M$. In realistic cases (see e. g. Eq. (92)), Eq. (64) is a good approximation because a power-law index $n$ depends only weakly on $M$.

We shall introduce for convenience the characteristic values of typical objects labeled by index ' $\Lambda$ ' and described by the condition $\sigma\left(t_{\Lambda}, M_{\Lambda}\right)=\delta_{c}$ at some fixed moment $t_{\Lambda}$ or redshift $z_{\Lambda}$. We can choose these quantities arbitrarily (because they will not enter into the final results), but satisfying the condition $z_{\Lambda} \gg\left(\Omega_{\Lambda} / \Omega_{m}\right)-1$, e. g. taking $z_{\Lambda} \sim 5-10$. The convenience of these normalizing values is that at $t<t_{\Lambda}$ the $\Lambda$-term can be neglected, while small-scale object formation occurs at these epochs. Let us introduce also the dimensionless mass $m$ as

$$
m=M / M_{\Lambda}
$$

Using the formation criterion (62) we obtain

$$
\delta\left(M, t_{f}\right)=\delta_{\mathrm{eq}} \frac{1+z_{\mathrm{eq}}}{1+z_{f}}=\delta_{c}
$$

because the growth-factor for the rising mode $D_{g}(t) \propto$ $(1+z) \propto t^{2 / 3}$ in the standard cosmological model at the matter dominated epoch. For a single clump with mass $M$ obeying $\delta_{\mathrm{eq}}=\nu \sigma_{\mathrm{eq}}(M)$ with an arbitrary $\nu$ one has

$$
\bar{\rho}_{\mathrm{int}}=\kappa \bar{\rho}\left(z_{f}\right)=\kappa \rho_{\mathrm{eq}}\left(\frac{1+z_{\mathrm{eq}}}{1+z_{f}}\right)^{3}=\kappa \rho_{\mathrm{eq}} \frac{\nu^{3} \sigma_{\mathrm{eq}}^{3}(M)}{\delta_{c}^{3}},
$$

and

$$
R=\left(\frac{3 M}{4 \pi \bar{\rho}_{\text {int }}}\right)^{1 / 3}=\nu^{-1} R_{\Lambda} m^{(n+5) / 6)},
$$

where $M$ and $R$ are respectively mass and radius of the clump, $\kappa=18 \pi^{2} \simeq 178$ and $R_{\Lambda}=\left(3 M / 4 \pi \kappa \bar{\rho}\left(t_{\Lambda}\right)\right)^{1 / 3}$. The value of $\kappa$ describes the clump density increasing during contraction and it can be found from Eq. (50) (see also [40]).

A number density of unconfined (free) clumps (i.e. of those not belonging to the more massive objects) is given by the Press-Schechter formulae [40]

$$
=\left(\frac{2}{\pi}\right)^{1 / 2} \frac{\rho}{M} \frac{\delta_{c}}{D_{g}(t) \sigma_{\mathrm{eq}}^{2}} \frac{d \sigma_{\mathrm{eq}}}{d M} \times \exp \left[\frac{-\delta_{c}^{2}}{2 D_{g}(t)^{2} \sigma_{\mathrm{eq}}^{2}}\right] d M,
$$

where the growth-factor $D_{g}(t)$ is normalized as $D_{g}\left(t_{\mathrm{eq}}\right)=$ 1. Let us consider the Press-Schechter distribution for the clumps hosted by the larger clump at the moment $t_{f}$ of its formation. Taking into account the density increase factor $\kappa$ we obtain

$$
\psi_{\mathrm{PS}}\left(t_{f}, m\right) d m=\kappa \phi_{\mathrm{PS}}\left(t_{f}, m\right) d m .
$$

As it will be demonstrated in this section the clumps are destroyed by tidal interaction and each clump has a small surviving probability $\xi<0.01$. A survived clump during its lifetime is surrounded by other clumps with distribution given by Eq. (69). When a host clump is destroyed, the survived clump finds itself hosted by larger clump with the small clump distribution inside given by the same Eq. (69) but with larger $t_{f}$. Since the tidal destruction is most effective at small distances, one should always consider the smallest possible host clump from the hierarchical structure, and the distribution of the small clumps around one at the consideration is always given by Eq. (69). The characteristic time is the time of formation $t_{f}$ of the smallest host clump, though time of destruction can be much larger than $t_{f}$.

The total energy (kinetic and potential) of a clump is given by

$$
|E|=\frac{3-\beta}{2(5-2 \beta)} \frac{G M^{2}}{R} .
$$

\section{B. The rate of internal energy growth}

Consider a host clump with mass $M_{h}$, radius $R_{h}$, and with the small clumps inside having distribution (69) and moving in the common gravitational potential with a velocity dispersion $\sim V_{h} \simeq G M_{h} / R_{h}$. Interacting tidally with its neighbors, a small clump increases its internal energy. We calculate first the rate of internal energy increase due to these interactions. The mass of the considered clump is $M=m M_{\Lambda}$, it is characterized by an arbitrary $\nu$ and its interaction with a target clump occurs at the impact parameter $l$. A target clump is characterized by mass $M^{\prime}$, radius $R^{\prime}$, radius of the core $R_{c}^{\prime}=x_{c} R$ with $x_{c} \sim 0.1$ and by universal density distribution (3).

An increase of internal energy of a clump $M$ during one fly-by in the momentum approximation [50] is given by:

$$
\Delta E=\frac{1}{2} \int d^{3} r \rho(r)\left(v_{x}-\tilde{v}_{x}\right)^{2},
$$

where $v_{x}$ is the velocity increase of DM particle in direction of axis $x$, and $\tilde{v}_{x}$ is the same for c. m. of the clump. The axis $x$ connects the c.m.'s of two clumps when the distance between them is the shortest. One approximately has

$$
v_{x}-\tilde{v}_{x} \simeq \frac{\partial v_{x}}{\partial l} \Delta l=\frac{\partial v_{x}}{\partial l} r \cos \psi,
$$

where $\psi$ is the polar angle in spherical coordinates.

For nearly straightforward propagation, the angle between $\vec{v}_{\text {rel }}$ and the line connecting c.m.'s of the clumps evolves as

$$
\frac{d \phi}{d t}=-\frac{v_{\mathrm{rel}}}{l} \cos ^{2} \phi,
$$


where $v_{\text {rel }} \simeq \sqrt{2} V_{h}$. Changing variable $t$ to $\phi$ in the Newton equation one gets

$$
\frac{d v_{x}}{d \phi}=-\frac{G M^{\prime}\left(r^{\prime}(\phi)\right)}{v_{\text {rel }} l} \cos \phi
$$

and after integration of this equation:

$$
v_{x}=\frac{2 G M^{\prime}}{v_{\text {rel }} R^{\prime}} g(y),
$$

where $y=l / R^{\prime}$,

$$
g(y)=\left\{\begin{array}{l}
y^{-1}, \quad y>1 ; \\
{\left[1+y^{3-\beta}\left(1-y^{2}\right)^{1 / 2}{ }_{2} F_{1}\left(\frac{3-\beta}{2}, \frac{1}{2}, \frac{3}{2}, 1-y^{2}\right)\right.} \\
\left.-\left(1-y^{2}\right)^{1 / 2}\right] / y, \quad y<1,
\end{array}\right.
$$

and ${ }_{2} F_{1}$ is the hypergeometric function.

The rate of internal energy growth due to collisions with all other clumps is

$$
\dot{E}=\int 2 \pi l v_{\mathrm{rel}} d l \int d M^{\prime} \psi\left(M^{\prime}, t\right) \Delta E .
$$

After simple calculations, one obtains

$$
\begin{aligned}
\dot{E}= & \frac{4 \pi(3-\beta)}{3(5-\beta)} \frac{G^{2} M R^{2}}{v_{\mathrm{rel}}} \int_{M}^{M_{h}} d M^{\prime} M^{\prime 2} \psi\left(M^{\prime}, t\right) \\
& \times\left[\frac{\lambda}{{R^{\prime}}^{2}}+\frac{1}{2}\left(\frac{1}{{R^{\prime}}^{2}}-\frac{1}{R_{h}^{2}}\right)\right],
\end{aligned}
$$

where

$$
\lambda=\int_{x_{c}^{\prime}}^{1} d y y\left(\frac{d g}{d y}\right)^{2}=0.11
$$

for $x_{c}^{\prime}=0.1$ and dependence of $\lambda$ on $x_{c}^{\prime}$ is very weak.

As the second process of tidal destruction we shall consider the interaction with the common gravitational potential of a host clump. Energy gain per mass unit at a distance $r$ from c. $m$. of the considered small clump $m$ during one periastron passage [50] is

$$
\left\langle E_{p}\right\rangle=\frac{G M_{h}}{R_{h}^{3}} r^{2}\left(\frac{R_{h}}{R_{p}}\right)^{\beta} \chi_{\mathrm{ecc}}(e) A(\omega \tau)
$$

where $e$ is the eccentricity, the function $\chi_{\text {ecc }}$ presented in [50], the adiabatic correction $A(x)=\left(1+x^{2}\right)^{-\gamma}$, $\gamma \simeq 2.5-3$ and $R_{p}$ is the periastron separation. The energy gain of a clump during one period $T_{\text {orb }}$ is $\Delta E=$ $\int\left\langle E_{p}\right\rangle \rho_{\text {int }}(r) d^{3} r$, and the rate of energy growth is

$$
\dot{E}=\frac{2 \Delta E}{T_{\text {orb }}} .
$$

The rate of energy growth due to the both of aforementioned processes is given by the sum of (78) and (81).
Using the distribution (69) in the integral of (78) and the total energy of a clump given by Eq. (70) we find:

$\frac{1}{T\left(m, m_{h}, \nu, \nu_{h}\right)} \equiv \frac{\dot{E}}{E} \simeq 2 t_{\Lambda}^{-1} \mu \nu_{h}^{9 / 2} \nu^{-3} m^{(n+3) / 2} m_{h}^{-3(n+3) / 4}$,

where

$$
\begin{aligned}
\mu= & \frac{2^{1 / 2} \kappa^{1 / 2}(5-2 \beta)}{3(5-\beta)}\left[\left(\frac{R_{h}}{R_{p}}\right)^{\beta} A(\omega \tau) \chi_{\mathrm{ecc}}(e)\right. \\
& \left.+\frac{1}{4 \pi^{1 / 2}}\left|\frac{n+3}{n+1}\right|\left(2 \lambda+\frac{n+5}{n+9}\right)\right] .
\end{aligned}
$$

The first term in the square brackets describes the interaction with common gravitational field of the host clump, while the second term — "collisions" with small clumps inside the host clump. Usually the former is larger than the latter. For calculations we shall consider an average orbit with $R_{h} / R_{p} \simeq 2$ and $T_{\text {orb }} \simeq 2 R_{h} / V_{h}$, and put $A(\omega \tau) \chi_{\text {ecc }}(e) \sim 1$. If to neglect the tidal interactions with the small clumps (the second term in the square brackets in Eq. (83) ) and to use $\beta=1.8$, one has $\mu \simeq 9.6$. The dependence of our final result (mass function of the clumps) on $\mu$ is weak, approximately as $\mu^{-1 / 3}$.

\section{Survival probability in the hierarchical model}

A small clump with mass $m$ during its life time can be a constituent part of many host clumps of successively larger masses $m^{\prime}$ and virial velocities $V^{\prime}$. After tidal disruption of the lightest host, a small clump automatically becomes a constituent part of the heavier host etc. Transition of a small clump from one host to another occurs almost continuously in time up to formation of a host where tidal destruction becomes inefficient. A fraction of small clumps with mass $m$ escaping the tidal destruction is given by $e^{-J}$, with

$$
J \simeq \sum_{m^{\prime}} \frac{\Delta t}{T\left(m, m^{\prime}, \nu, \nu^{\prime}\right)}
$$

where summation goes over the intermediate big clumps which successively host the small clump $m$, and $\Delta t$ approximately equals to the difference of formation times of two successive hosts.

Let us introduce the notation: $m_{1}$ is the mass of the first (lightest) host clump which contains the considered light clump $m$, and $m_{n}$ is the last such object, e. g. the Galactic halo. A formation epoch for the host clump $m^{\prime}$ is

$$
t_{f}\left(m^{\prime}, \nu^{\prime}\right)=t_{\Lambda}\left(\frac{1+z^{\prime}}{1+z_{\Lambda}}\right)^{-3 / 2}=t_{\Lambda} m^{\prime}(n+3) / 4 \nu^{\prime}-3 / 2
$$

Note that $J$ does not depend on $t_{\Lambda}$ since it enters linearly in $t_{f}$ and in $T$ as seen from Eq. (82). 
The first host gives a major contribution to the clump destruction, especially if its mass $m_{1}$ is close to $m$ and $\nu_{1} \simeq \nu$. For the considered clump $m$ and for the first two hosts $\sigma_{\text {eq }}(M) \simeq \sigma_{\text {eq }}\left(M_{1}\right) \simeq \sigma_{\text {eq }}\left(M_{2}\right)$ because of $\sigma_{\text {eq }}(M)$ depends weakly on $M$ according to (64). The considered clump $m$ and the first two hosts differ mainly by masses and by values of $\nu\left(\nu \geq \nu_{1} \geq \nu_{2}\right)$. It justifies the following simplification: we consider all hosts beginning from the second one as the typical objects $\left(\nu_{i}=1\right.$ for $\left.i=2,3, \ldots\right)$ and separating the first term in the sum (84), substitute the remaining sum by the integral.

$$
\begin{aligned}
J & \simeq \frac{t_{f}\left(m_{2}, \nu_{2}=1\right)-t_{f}\left(m_{1}, \nu_{1}\right)}{T\left(m, m_{1}, \nu, \nu_{1}\right)} \\
& +\int_{m_{2}}^{m_{n}} \frac{1}{T\left(m, m^{\prime}, \nu, \nu^{\prime}=1\right)} \frac{d t_{f}\left(m^{\prime}, \nu^{\prime}=1\right)}{d m^{\prime}} d m^{\prime}
\end{aligned}
$$

We may change now the lower limit $m_{2}$ to $m_{1}$ in the above integral because it depends on $m_{2}$ weakly, only through $m_{2}^{(n+3) / 2}$ with $(n+3) / 2 \ll 1$, where $n$ is given by Eq. (64). In addition we may put the upper limit $m_{n} \rightarrow$ $\infty$ without loss of accuracy. Inserting Eq. (82) into (86) we obtain finally the following approximate expression for $J$ :

$$
\begin{aligned}
J & \simeq 2 \mu \frac{\nu_{1}^{9 / 2}}{\nu^{3}}\left(\frac{m}{m_{1}}\right)^{(n+3) / 2}\left(1-\nu_{1}^{-3 / 2}\right) \theta\left(\nu_{1}-1\right) \\
& +\mu \nu^{-3}\left(\frac{m}{m_{1}}\right)^{(n+3) / 2},
\end{aligned}
$$

where the step-function $\theta(x)=1$ for $x>0$ and $\theta(x)=0$ for $x \leq 0$. In the case of $\nu_{1}=1$ the formation moment of the first host almost coincides with the formation moment of the second one, for which $\nu_{2}=1$ in the used approach.

The differential fraction of mass in the form of clumps which escape the tidal destruction in the hierarchical objects can be found as

$$
\begin{aligned}
\xi(n, \nu) \frac{d m}{m} d \nu= & d m d \nu(2 / \pi) e^{-\nu^{2} / 2} \int_{0}^{\nu} d \nu_{1} e^{-\nu_{1}^{2} / 2} \\
& \times \int_{t_{f}(m, \nu)}^{\infty} d t_{1}\left|\frac{\partial^{2} F\left(m, t_{1}\right)}{\partial m \partial t_{1}}\right| e^{-J},
\end{aligned}
$$

where the variable $m_{1}$ in $J$ is connected with $t_{1}$ and $\nu_{1}$ according to Eq. [85), $F(m, t)$ is the mass fraction of unconfined clumps with masses smaller than $m$ at the moment $t$, which according to [40] is given by

$$
F(m, t)=\operatorname{erf}\left\{\frac{\delta_{c}}{\sqrt{2} \sigma_{\mathrm{eq}}(M) D_{g}(t)}\right\} .
$$

Here $\operatorname{erf}(\mathrm{x})$ is the error-integral and $D_{g}(t)$ is the growthfactor. After numerical calculations for $\beta=1.8$ we finally obtain:

$$
\xi(n, \nu) \simeq(2 \pi)^{-1 / 2} e^{-\nu^{2} / 2}(n+3) y(\nu),
$$

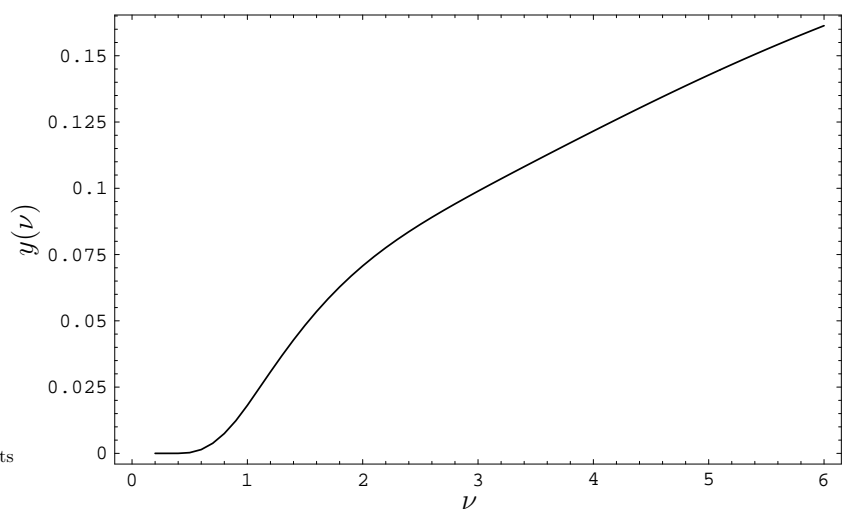

FIG. 2: Function $y(\nu)$ from Eq. (90) obtained by the numerical integration of (88). This curve is valid with good accuracy for all $\beta$ from the interval $1 \leq \beta \leq 2$.

where the function $y(\nu)$ is plotted in the Fig. 2 It weakly depends on $\beta$.

This is the main result of this section. We shall refer to $\xi$ as to the clump survival probability.

Our final result, the distribution of surviving clumps over their masses $M$ and $\nu$, which for the fixed $M$ determines the clump radius $R$, is given by $\xi(n, \nu) d \nu d M / M$, where $\xi$ depends very weakly on $M$ only through the weak dependence of $n(M)$ (see Eq. (64)). For most numerous clumps with $\nu=1$ and for the unit intervals $d \nu$ and $d \ln M, \xi$ has a meaning of fraction of DM mass in the form of clumps relative to free DM particles, as it was introduced in Eq. (10). By integrating over $\nu$, we obtain

$$
\xi_{\text {int }} \simeq 0.01(n+3)
$$

This means that, for different $n$, about $0.1-0.5 \%$ of clumps survive the stage of tidal destruction in each logarithmic mass interval $\Delta \ln M \sim 1$.

Several remarks are in order.

The physical meaning of the survived clump distribution $\xi(n, \nu) d \nu d M / M$ is different from that for unconfined (free) clumps given by the Press-Schechter mass function $\partial F / \partial m$. Surviving clump distribution implies that each DM particle belongs simultaneously to several host clumps put into each other, and by this reason the integral $\int \xi d m / m$ is divergent.

To calculate $\xi$ we need to know the power-law index $n$ in the perturbation spectrum, which can be taken as $n_{\text {eff }}$ from Eq. (64) for given $\sigma_{\text {eq }}(M)$. To find the latter, the primeval (e. g. inflation) power spectrum of fluctuation is needed. The simplest inflation models give $P_{p}(k) \propto k^{n_{p}}$ with $n_{p} \approx 1$. The analysis of the WMAP measurement of CMB anisotropy [51] gives power law spectrum with $n_{p}=$ $0.99 \pm 0.04$ in good agreement with $n_{p}=1$. However, when data from $2 \mathrm{dF}$ galaxy power spectrum and Ly- $\alpha$ are included in analysis, the best-fit favors a mild tilt, $n_{p}=0.96 \pm 0.02$.

The variance $\sigma_{\text {eq }}(M)=\sigma_{(0)}$ from Eq. (44) in the small scale range is found in [52] (see also [53]). We present 
this result as

$$
\begin{aligned}
\sigma_{\mathrm{eq}}(M) & \simeq \frac{2 \times 10^{-4}}{\sqrt{f_{s}\left(\Omega_{\Lambda}\right)}}\left[\ln \left(\frac{k}{k_{\mathrm{eq}}}\right)\right]^{3 / 2}\left(\frac{k}{k_{h 0}}\right)^{\left(n_{p}-1\right) / 2} \\
& \simeq 8.2 \times 10^{3.7\left(n_{p}-1\right)-3}\left[1-0.06 \lg \left(\frac{M}{M_{\odot}}\right)\right]^{3 / 2} \\
& \times\left(\frac{M}{M_{\odot}}\right)^{\left(1-n_{p}\right) / 6}
\end{aligned}
$$

where $k_{\text {eq }}$ and $k_{h 0}$ correspond to mass inside the cosmological horizon at the moments $t_{\mathrm{eq}}$ and $t_{0}$, respectively, and

$$
f_{s}\left(\Omega_{\Lambda}\right)=1.04-0.82 \Omega_{\Lambda}+2 \Omega_{\Lambda}^{2}
$$

according to [52]. We used above the relation $k \propto M^{-1 / 3}$ and the values

$$
M_{\mathrm{eq}}=1.5 \times 10^{49} \Omega_{m}^{-2} h^{-4} \mathrm{~g}, \quad M_{h 0}=6 \times 10^{55} \Omega_{m} h^{-1} \mathrm{~g} .
$$

Using the power-law spectrum of fluctuations down to the small scales, while normalization by the CMB anisotropy is performed at large scale, implies an extrapolation of the spectrum by many orders of magnitudes. Such extrapolation is justified only by the confidence to inflation models which predict the power-law spectrum valid for many orders of mass magnitudes.

It is interesting to note that the differential number density of clumps in the Galactic halo $n(M) d M \propto$ $d M / M^{2}$, obtained from Eq. (90), is very close (including the normalization coefficient) to that obtained in the numerical simulations for clumps with large masses $M \geq 10^{8} M_{\odot}\left[n(M) d M \propto d M / M^{1.9}[4]\right]$. Strictly speaking our calculations are not valid for clumps with these masses, because of their destruction in the halo up to present epoch $t_{0}$ and accretion of new clumps to the halo. Nevertheless, for the small interval of masses where the power-law spectrum can be used as a rather good approximation, our approach can be roughly valid.

\section{NUMERICAL RESULTS}

In this section we shall present the numerical results of our calculations: enhancement factor for annihilation signal, the distribution of DM clumps over their masses $M$ and radii $R$, and the distribution of clumps in the galactic halo.

Using Eqs. (6) and (7) we find the enhancement of the annihilation signal due to clumpiness of the halo as generalization of Eq. (10) for the clumps distributed over $M$ and $R$ :

$$
\eta\left(M_{\min }, n_{p}\right)=1+\frac{1}{\tilde{\rho}_{\mathrm{DM}}} \int_{\nu_{\min }}^{\infty} d \nu \int_{M_{\min }} \frac{d M}{M} S\left[\beta, x_{c}(\nu)\right] \xi \bar{\rho}_{\mathrm{int}}(M, \nu),
$$

where

$$
\xi=\xi(n, \nu)
$$

is defined by Eq. (90), an effective spectrum index $n\left(M, n_{p}\right)$ is calculated from Eq. (64) for primeval (inflation) spectrum index $n_{p}$, with $\sigma_{\text {eff }}(M)$ taken in the form (92); $\bar{\rho}_{\text {int }}$ is given by Eq. (66) and $\nu_{\text {min }} \simeq 0.55$. Function $S$ is taken in the form (4), which corresponds to the density profile (3) and we used Eq. (60) for a clump virial radius $R$.

The major part of the survived clumps are formed from fluctuations with a mean value of the peak height

$$
\langle\nu\rangle \simeq \frac{\int d \nu \nu e^{-\nu^{2} / 2} y(\nu)}{\int d \nu e^{-\nu^{2} / 2} y(\nu)} \simeq 1.6 .
$$

Meanwhile the main contribution to the enhancement of the annihilation signal (95) comes from the clumps with an effective value of the peak height:

$$
\langle\nu\rangle_{\mathrm{ann}} \simeq \frac{\iint d \nu \nu S \xi \bar{\rho}_{\mathrm{int}} d M / M}{\iint d \nu S \xi \bar{\rho}_{\mathrm{int}} d M / M} \simeq 2.5
$$

for $\beta=1.8$. The clumps with $\nu \simeq 2.5$ have $x_{c} \simeq 0.05$. 10]:

For the Galactic halo we use the NFW density profile

$$
\rho_{\mathrm{DM}}(l)=\frac{\rho_{0}}{(l / L)(1+l / L))^{2}},
$$

with $L=45 \mathrm{kpc}$ according to [54], and $\rho_{0}$ fixed by the local density value $\rho_{\mathrm{DM}}\left(r_{\odot}\right)=0.3 \mathrm{GeV} \mathrm{cm}^{-3}$. With these parameters the halo mass within the virial radius of $100 \mathrm{kpc}$ is $10^{12} M_{\odot}$. Eq. (9) gives $\tilde{\rho}_{H}=1.02 \rho_{H}\left(r_{\odot}\right)$, i. e. these values practically coincide.

The values of global enhancement $\eta\left(M_{\min }, n_{p}\right)$ as given by Eq. (95) are displayed in Figs. 3 15 for different values of $M_{\min }, \beta$ and $n_{p}$. As a representative example consider the clump with the Gurevich-Zybin [9] density profile with $\beta=1.8$ (see Fig. 5) : numerically $\eta=5$ for $M_{\min }=$ $2 \times 10^{-8} M_{\odot}$ and $n_{p}=1.0$. It strongly increases at smaller $M_{\min }$ and larger $n_{p}$. For example, for $n_{p}=1.1$ and $n_{p}=1.2$ at the same $M_{\min }=2 \times 10^{-8} M_{\odot}$, enhancement becomes tremendously large, $\eta=130$ and $\eta=4 \times 10^{3}$, respectively.

Our approach is based on the hierarchical clustering model in which smaller mass objects are formed earlier than the larger ones, i. e. $\sigma_{\text {eq }}(M)$ diminishes with the growing of $M$. This condition is satisfied for objects with mass $M>M_{\min } \simeq 2 \times 10^{-8} M_{\odot}$ only if the primordial power spectrum has the value of the power index $n_{p}>0.84$. As seen from Figs. 3] [5] in this case the enhancement of the annihilation signal in fact is absent, $\eta \simeq 1$, for $n_{p}<0.9$. 


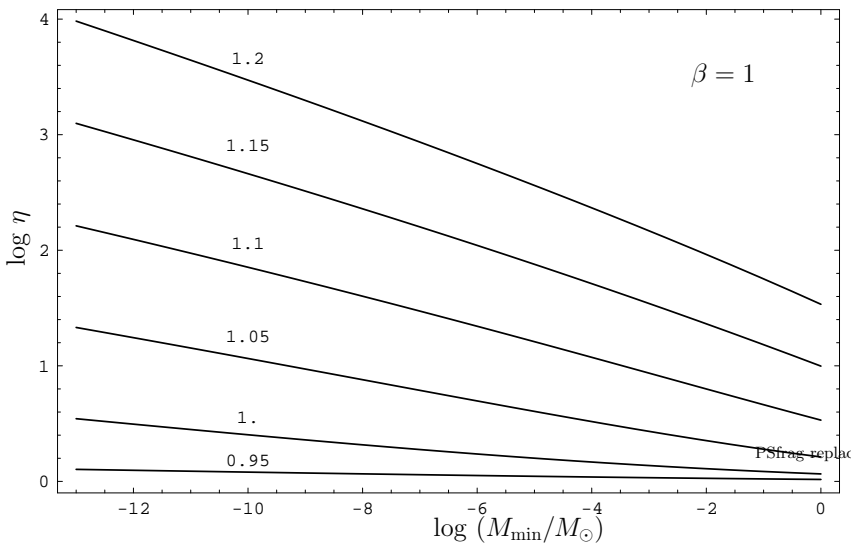

FIG. 3: The global enhancement $\eta$ of the annihilation signal from Eq. 95 as a function of the minimal clump mass $M_{\min }$, for clump density profile with index $\beta=1$ and for different indices $n_{p}$ of primeval perturbation spectrum. The curves are marked by the values of $n_{p}$.

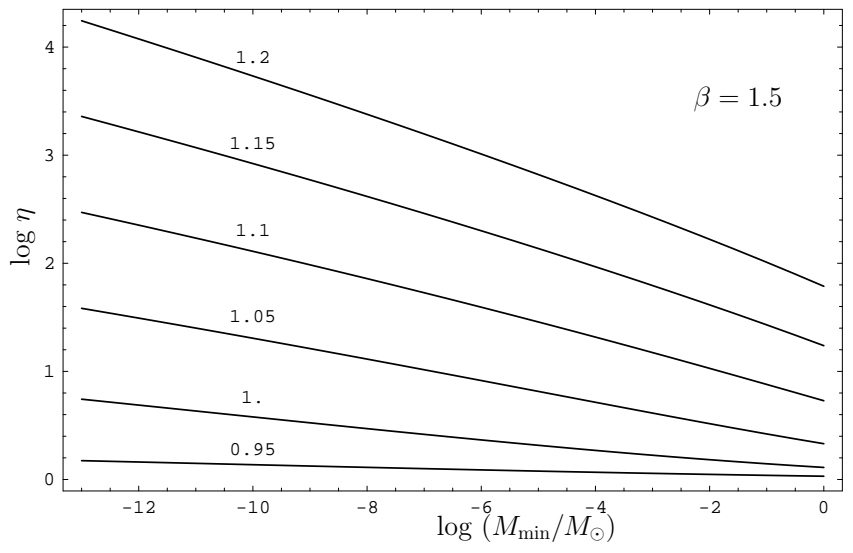

FIG. 4: The same as Fig. 3 but for $\beta=1.5$.

We discussed above the global enhancement of the annihilation signal. In fact the enhancement varies in the different directions relative to the Galactic Center (GC). It can be easily seen from Eqs. (6) and (7), which show that while $I_{\mathrm{cl}}$ is proportional to $n_{\mathrm{cl}}(l)$ (and thus to $\rho_{\mathrm{DM}}(l)$ everywhere except the core), $I_{\text {hom }}$ is proportional to $\rho_{\mathrm{DM}}^{2}(l)$. It implies that relative contribution of $I_{\text {hom }}$ increases in the directions close to GC where $\rho_{\mathrm{DM}}(l)$ is larger. This effect is further enhanced by the destruction of the clumps in the core around the GC. Our numerical calculations confirm this expectation: the ratio of enhancement in the directions to GC and Anticenter is 0.2 for NFW density profile and for the core radius $3 \mathrm{kpc}$. Therefore, the presence of the clumps diminishes the anisotropy of the annihilation signal, caused by the density profile of DM in the halo.

Our results suffer from uncertainties in input parameters. As was mentioned above $\tilde{\rho}_{\mathrm{DM}} \simeq \rho_{\mathrm{DM}}\left(r_{\odot}\right)$. It remains approximately true not only for the NFW density profile but also for other profiles discussed in the

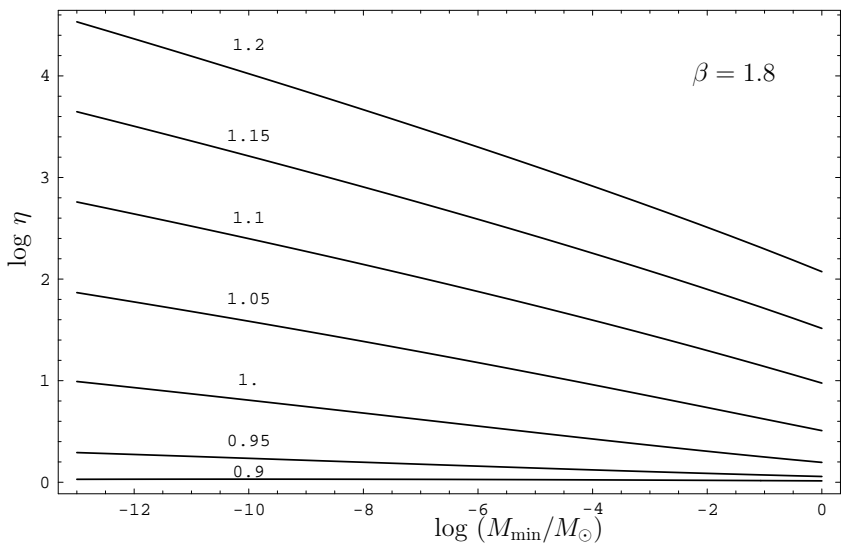

FIG. 5: The same as Fig. [3 but for $\beta=1.8$.

literature. For example, for isothermal profile with the core radius $10 \mathrm{kpc}, \tilde{\rho}_{\mathrm{DM}}=0.65 \rho_{\mathrm{DM}}\left(r_{\odot}\right)$. Another uncertainty in the value of $\eta$ is imposed by value of $\rho_{\mathrm{DM}}\left(r_{\odot}\right)$. According to different estimates $\rho_{\mathrm{DM}}\left(r_{\odot}\right)=$ $(0.2-0.6) \mathrm{GeV} \mathrm{cm}^{-3}$. The corresponding uncertainty in $\eta$ is given by factor $0.5-1.5$.

For illustration we shall describe numerically the properties of clumps which give the main contribution to annihilation rate. Basically, they are those with $M \sim M_{\min }$ and $\nu \sim\langle\nu\rangle$. The r.m.s. fluctuation values $\sigma_{\text {eq }}(M)$ for clumps with minimal mass $M_{\min } \simeq 2 \times 10^{-8} M_{\odot}$ and for $n_{p}=1$ and 1.2 are $\sigma_{\text {eq }}=0.015$ and 0.14 , respectively, according to Eq. (92). From Eq. (98) the effective value of $\nu=\delta_{\text {eq }} / \sigma_{\text {eq }}$ is $\langle\nu\rangle \simeq 2.5$. From Eqs. (66), 67 follows that clumps with this $\nu$ are characterized by the density and radius $\bar{\rho}_{\text {int }} \simeq 2 \times 10^{-22} \mathrm{~g} \mathrm{~cm}^{-3}, R \simeq 3.6 \times 10^{15} \mathrm{~cm}$ and $\bar{\rho}_{\text {int }} \simeq 2 \times 10^{-19} \mathrm{~g} \mathrm{~cm}^{-3}, R \simeq 3.7 \times 10^{14} \mathrm{~cm}$ for $n_{p}=1$ and 1.2 , respectively. The part of Galactic halo mass in the form of these clumps is of the order of $\xi_{\text {int }} \sim 0.002$ according to Eq. (91). A mean number density of the clumps in the halo is $\sim 25 \mathrm{pc}^{-3}$.

We have given above the characteristic values for clumps with dominant contribution to the annihilation signal. The general distribution of clumps in the Galactic halo can be readily calculated numerically from Eq. (90), changing (for given $M$ ) the distribution over $\nu$ by that over $d R$ :

$$
n_{\mathrm{cl}}(M, R) d \ln M d \ln R=\frac{\rho_{\mathrm{DM}}\left(r_{\odot}\right)}{M} \xi(M, \nu) d \ln M d \nu
$$

Note that definition of the clump number density $n_{\mathrm{cl}}$ here does not coincides with the similar one in Eq. (5) where $d N$ is given per $d R$ and $d M$.

The distribution $M n_{c l}(M, R)$ is presented in Fig. 6] as function of $R$ for different $M$ and for distance $8.5 \mathrm{kpc}$ from the Galactic center.

The radius of a clump $R$ in most general case is determined by $M$ and $\nu$. Due to weak dependence of $\sigma_{\text {eq }}(M)$ on $M$, the radius of the clump $R(M)$ with sufficiently good accuracy is proportional to $M^{1 / 3}$. From Eqs. (66) 


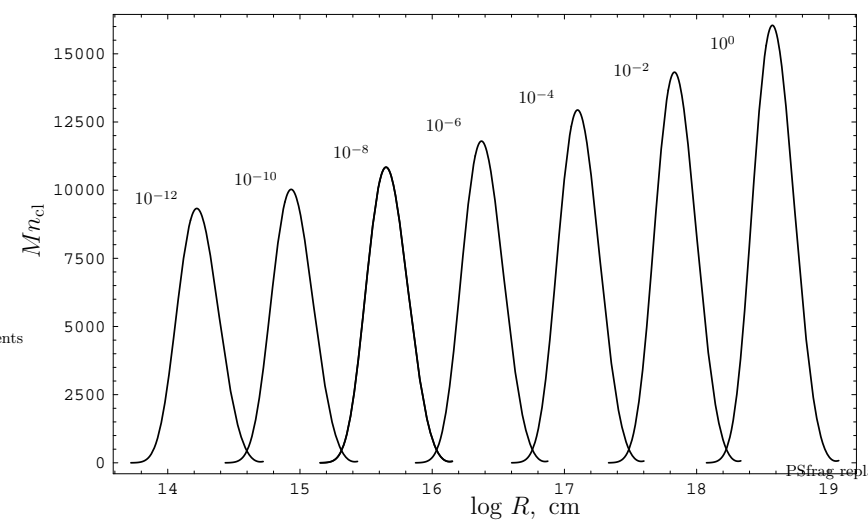

FIG. 6: The mass density of clumps in the Galactic halo $M n_{c l}(M, R)$ in units $M_{\odot} / \mathrm{kpc}^{3}$, from Eq. (100), as function of their radius $R$ at the distance $8.5 \mathrm{kpc}$ from the Galactic center for $n_{p}=1.0$. The curves are labeled by values of clump masses in $M_{\odot}$

and (67) we have

$$
R \simeq 1.5 \times 10^{16}\left(\frac{M}{10^{-6} M_{\odot}}\right)^{1 / 3}\left(\frac{\nu}{2.5}\right)^{-1} \mathrm{~cm},
$$

for $n_{p}=1.0$. For $n_{p}=1.1$ the numerical factor in the Eq. (101) is $5.5 \times 10^{15} \mathrm{~cm}$.

How clumps are distributed in the Galactic halo? One may expect that this distribution is the same as distribution of the free DM particles in the halo. This is true for large distances $l$ from Galactic Center, while at small $l$ the tidal interaction with stars results in the destruction of small clumps and in the formation of the core with radius $L_{c}$ in the clump distribution. The destruction of a clump propagating in the space filled by stars has been studied in [9]. The destruction is important only for clumps inside the bulge, i.e. at the distance $l \leq 3 \mathrm{kpc}$ from the Galactic Center. Clumps outside the bulge at the distances $3 \mathrm{kpc} \leq l \leq 10 \mathrm{kpc}$ can interact tidally with stars in the disk. But time of crossing the disk is very small (in comparison with orbital period) and this process is not important.

The number density of clumps outside the bulge is proportional to the halo density, e. g. to $\xi \rho_{\mathrm{DM}}(l)$ in the case of the NFW distribution given by Eq. (99), or can be obtained from (99) and Fig. [6 by simple scaling.

The density distribution for stars in the bulge according to [55] is given by

$$
\rho_{s}(l)= \begin{cases}\tilde{\rho}(l / \tilde{r})^{-1.8}, & l<\tilde{r}, \\ \tilde{\rho}(l / \tilde{r})^{-3}, & l>\tilde{r},\end{cases}
$$

where $\tilde{\rho}=1.8 M_{\odot} / \mathrm{pc}^{3}$ and $\tilde{r}=800 \mathrm{pc}$. From Eq. (46) of 2] by substituting Eq. (102) we obtain that inside the bulge $(l \leq 3 \mathrm{kpc})$, the clumps with $M \leq 10^{-4} M_{\odot}$ are destructed during the Hubble time. Thus, for these masses the core radius $L_{c}$ coincides with the size of the bulge $L_{\text {bulge }} \simeq 3 \mathrm{kpc}$.

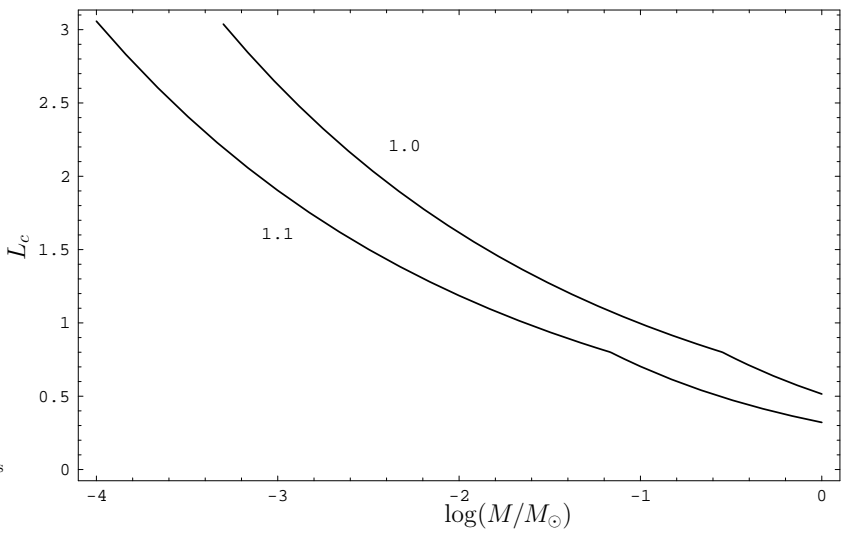

FIG. 7: The radius $L_{c}$ of the Galactic core (in kpc) in the distribution of clumps with masses $M$ for $n_{p}=1.0$ and $n_{p}=$ 1.1 in the case $\nu=2.5$.

Clumps with $M \geq 10^{-4} M_{\odot}$ are destructed during the Hubble time within distances from the Galactic Center shown in Fig. 7 for $n_{p}=1.0, n_{p}=1.1$ and $\nu=2.5$. This distance defines the radius of the core $L_{c}$ for clumps of the given mass $M$.

Our calculations for enhancement of annihilation signal disagree with those in $16,27,28]$.

In 16] the singularity in the Galactic Center is cut at very small core radius, which results in too strong annihilation signal. According to our calculations the radius of the core is much larger, and the distribution of the clumps in the halo also has a core.

In 27, 28 the large enhancement of the signal is found for heavy clumps with $M>10^{6} M_{\odot}$ [27] and $M>10^{2} M_{\odot}$ [28]. If it were true, the total signal from clumps with $M \geq M_{\min }$ would be too large. Too small core radius was used in these calculations, too.

\section{CONCLUSIONS}

We have calculated the number density of the smallscale clumps in the Galactic halo and their distribution over masses $M$, radii $R$ and distances to the Galactic Center in the framework of the standard cosmological model with the primeval density perturbation $P(k) \propto$ $k^{n_{p}}$ taken from the inflation models with $n_{p} \simeq 1$ (the Harrison-Zeldovich spectrum). The most important element of our calculations is inclusion of the tidal interactions, which result in the formation of the clump core and destruction of small-scale clumps.

We consider most conservative case of the Gaussian adiabatic fluctuations which enter the non-linear stage of evolution, $t_{\mathrm{nl}}$, at the matter-dominated epoch $t_{\mathrm{nl}}>t_{\mathrm{eq}}$, where $t_{\mathrm{eq}}$ is the moment of equality. The time of smallscale clump formation $t_{f}$ for a clump with mass $M$ is given by two equation: formation criterion $\delta\left(M, t_{f}\right)=\delta_{c}$ and by height of peak density of a fluctuation in units of dispersion $\nu=\delta_{\text {eq }} / \sigma_{\text {eq }}(M)$, taken at the epoch $t_{\text {eq }}$ [see 
Eqs. (48) and (62) for explanation and notation]. All processes we are interested in, take place at $t \geq t_{\mathrm{eq}}$ at the stage of non-linear evolution. The growth of fluctuations in the non-linear regime we study in the framework of the Press-Schechter theory of hierarchical clustering with the tidal interactions included as the new element. The picture of hierarchical clustering and clump destruction can be described in the following way. The clumps of the minimal mass are formed first. A clump of larger mass, which hosts the smaller clumps, is formed later. A bigger clump, which includes the considered hosts with their content, is formed further later etc. The clumps are destroyed in tidal interactions with other small clumps and by gravitational field of a host clump, with the former process being subdominant. The calculated mass density of the survival clumps, $\xi(M, \nu) d \nu d M / M$, with mass $M$ and $\nu$, is given by Eq. (90), with the survival probability being typically $\xi \sim 0.001-0.005$. The clump number density in the Galactic halo $n_{\mathrm{cl}}(M, R)$ for the clumps with mass $M$ and radius $R$ is shown in Fig. 6] These clumps are distributed in the Galactic halo as function of a distance to the Galactic Center $l$. At large distance the distribution must be the same as found in the numerical simulations (e. g. the NFW profile). At small distance there is a core produced by tidal interaction of the clumps with the stars in the bulge. The radius of the core, $L_{c}$ is given in Fig. 7 at $M \geq 10^{-4} M_{\odot}$ and it is equal to the radius of the bulge $L_{c} \sim 3 \mathrm{kpc}$ for smaller clump masses.

The mass spectrum of the clumps is characterized by a cutoff at $M_{\text {min. }}$. Its value depends on the properties of the DM particle, and thus it is model dependent. The existing calculations of $M_{\text {min }}$ differ drastically: from $M_{\min } \sim 10^{-12} M_{\odot}[42]$ to $M_{\min } \sim 10^{-7} M_{\odot}$ [43].

Cold Dark Matter particles at high temperature $T>$ $T_{f} \sim 0.05 m_{\chi}$ are in thermodynamical (chemical) equilibrium with cosmic plasma, when their number density is determined by temperature. After decoupling at $t>t_{f}$ and $T<T_{f}$, the DM particles remain for some time in the kinetic equilibrium with plasma, when temperature of CDM particles $T_{\chi}$ is equal to temperature of plasma $T$, but number density $n_{\chi}$ is not Planckian any more. At this stage the CDM particles are not perfectly coupled to the cosmic plasma. Collisions between a CDM particle and fast particles of ambient plasma result in exchange of momenta and a CDM particle diffuses in a space. Due to diffusion, the DM particles leak from the small-scale fluctuations, and thus their distribution obtain a cutoff at minimal mass $M_{D}$. The diffusion coefficient is determined by elastic scattering of DM particles off the plasma particles. Our calculations made for neutralino, for which we have chosen the pure bino state, give

$$
M_{\mathrm{D}}=4.3 \times 10^{-13}\left(\frac{m_{\chi}}{100 \mathrm{GeV}}\right)^{-15 / 8}\left(\frac{\tilde{M}}{1 \mathrm{TeV}}\right)^{-3 / 2} M_{\odot},
$$

where $m_{\chi}$ is the neutralino mass and $\tilde{M}$ is (approximately) the mass of sneutrino and selectron, which are assumed to be equal. The functional dependence of
Eq. (25) and numerical value of Eq. (26) obtained in the diffusion approximation coincide with the corresponding results obtained by different method in 42 .

When the energy relaxation time for DM particles, $\tau_{\text {rel }}$, becomes larger than the Hubble time $H^{-1}(t)$, DM particles get out of kinetic equilibrium. This conditions determines the time of kinetic decoupling $t_{d}$. At $t \geq t_{d} \mathrm{CDM}$ matter particles are moving in free streaming regime and all fluctuations on the free-streaming scale $\lambda_{f s}$ and less are washed out. In contrast to [43], we have calculated the free-streaming length $\lambda_{f s}$ taking into account the distribution of neutralino (bino) velocities over absolute values and angles from radial directions. The cosmological expansion in the vicinity of $t_{\mathrm{eq}}$ is taken exactly, without usual step-function approximation. Our value of $M_{\min }$ due to free-streaming effect is

$M_{\min }=1.5 \times 10^{-8}\left(\frac{m_{\chi}}{100 \mathrm{GeV}}\right)^{-15 / 8}\left(\frac{\tilde{M}}{1 \mathrm{TeV}}\right)^{-3 / 2} M_{\odot} ;$

see Eq. (38) for more parameters involved.

When normalized to the same masses of neutralino and sleptons, our value of $M_{\min }$ coincides only by order of magnitude with 43 .

The evolution of a density fluctuation in the non-linear regime results in the density profile of a clump. The analytic theory of this phenomenon was developed by Gurevich and Zybin (for a review see 9] ), for the numerical simulations see [3, 10]. The initial single-stream flow leads to formation of initial singularity. In contrast to energy-dissipating matter (e. g., baryons), in the flow of non-dissipative matter the multistream instability develops [9], when at one point several streams with different radial velocities exist. The surfaces with different number of streams are separated by caustics, which number increases rapidly towards the center. The matter is gravitationally captured in a such structure. The density singularity is produced in the center, unless the additional phenomena are included in the consideration. As such the interaction with the damped mode 9] and annihilation of DM particles [23] were previously studied. We have demonstrated here that tidal forces due to external gravitational field cause the deflection of DM particles from radial motion, and prevent thus formation of singularity. The produced core has a radius $R_{c}$ given in the approximate form as

$$
x_{c}=\frac{R_{c}}{R} \simeq 0.3 \nu^{-2},
$$

(see Eq. (60) for the exact expression and the discussion afterwards). This radius is much bigger than those obtained in 9 , 23].

The majority of clumps are formed from $\nu \sim 1$ peaks, while the survived clumps are characterized on average by $\nu \simeq 1.6$. The clumps which give the dominant contribution to the annihilation signal have $\nu \simeq 2.5$.

In spite of small surviving probability, $\xi \sim 0.1-0.5 \%$, clumps in most cases provide the dominant contribution 
to the annihilation rate in the halo. The enhancement of the annihilation signal can be characterized by ratio $\eta=\left(I_{\mathrm{cl}}+I_{\mathrm{hom}}\right) / I_{\mathrm{hom}}$, where $I_{\mathrm{cl}}$ is the annihilation signal from the clumps, and $I_{\text {hom }}$ - from homogeneously distributed DM particles with the NFW density profile in the Galactic halo. The main contribution to $\eta$ is given by $\nu \simeq 2.5$ and $M \simeq M_{\min }$. The signal enhancement $\eta$ is shown numerically in Figs. 3 [5 One can see that practically for all allowed values of primeval perturbation spectrum index $n_{p} \geq 1.0$ the annihilation signal from clumps gives the dominant contribution. This result does not depend on the properties of DM particles.

The observations favor the spectrum index $n_{p}=1.0$ 51]. The enhancement of the annihilation signal for this value of $n_{p}$ is described by factor $2-5$ for different $\beta$ with uncertainties due to values of $M_{\min }$ and other parameters.

The clumps which give the dominant contribution to the annihilation signal have approximately the following properties in the case $n_{p}=1$ : The mass $M \sim M_{\min }$ and $\nu \sim 2.5$, the radius $R \simeq 3.6 \times 10^{15} \mathrm{~cm}$ and the radius of the core $R_{c} \simeq 1.8 \times 10^{14} \mathrm{~cm}$, the mean internal density of the clump $\bar{\rho}_{\text {int }} \simeq 2.5 \times 10^{-22} \mathrm{~g} \mathrm{~cm}^{-3}$, the fraction of the halo mass in the form of these clumps $\xi_{\text {int }} \sim 0.002$, and the mean number density of these clumps in the halo $n_{\mathrm{cl}} \sim 25 \mathrm{pc}^{-3}$.

Recently HEAT collaboration detected excessive flux of cosmic ray positrons at energy $E \sim 10 \mathrm{GeV} 56$. According to [57], if this positron flux is produced by annihilation of neutralinos the enhancement factor of order of 30 is needed. The calculations presented here show that such enhancement can be reached in the considered scenario in case of extreme combination of parameters.

\section{Acknowledgments}

This work has been supported in part by the INTAS grant No. 99-1065; V.D. and Yu.E. are supported also by the RFBR grants No. 03-02-16436-a and No. 01-0217829 .

\section{APPENDIX A: CROSS SECTIONS OF NEUTRALINO SCATTERING OFF ELECTRONS AND NEUTRINOS}

As neutralino we shall consider here a pure bino $(\chi=$ $\tilde{B})$. The Lagrangian for interaction of bino with left and right components of a fermion $f$ can be written (see e. g. [58, [59]) as

$$
\begin{aligned}
\mathcal{L}_{f \tilde{f} \chi}= & -g \sqrt{2} \tan \theta_{\mathrm{W}}\left(e_{f}-T_{3 f}^{L}\right) \bar{f} P_{R} \chi \tilde{f}_{L} \\
& +g \sqrt{2} \tan \theta_{\mathrm{W}} e_{f} \bar{f} P_{L} \chi \tilde{f}_{R},
\end{aligned}
$$

where $g$ is $\mathrm{SU}(2)$ coupling constant, $\theta_{\mathrm{W}}$ is the Weinberg angle $\left(\sin ^{2} \theta_{\mathrm{W}}=0.231\right), e_{f}$ is electric charge of the fermion $f$ in the units of electron charge, $T_{3 f}^{L}$ is the projection of weak isospin for $f_{L}, P_{R}=1 / 2\left(1+\gamma_{5}\right)$ is a projection operator which cuts the left component from the operator $\bar{f}$ in Eq. (A1); $\tilde{f}_{L}$ is the left sfermion. The first term in the Lagrangian (A1) is $\mathcal{L}_{f_{L} \tilde{f}_{L} \chi}$, the second $\mathcal{L}_{f_{R} \tilde{f}_{R} \chi}$. When $f$ is ultra-relativistic in the frame where neutralino is at rest, there is no interference for scattering of the left and right components of the fermions (the interference terms are proportional to $m_{f}$ ). Therefore, we shall calculate $f \chi$ cross-section for left $f_{L}$ and right $f_{R}$ fermions separately.

Scattering of the left fermion with $e_{f}=-1$ and $T_{3 f}=$ $-1 / 2$ (e. g. $e, \mu, \tau$ ) off bino are described by the two diagrams in $s$ - and $u$-channels as shown below

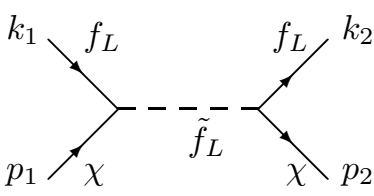

$S$

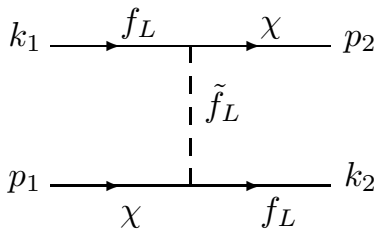

$u$
The standard calculations for matrix elements give for $|M|^{2}=\left|M_{s}\right|^{2}+\left|M_{u}\right|^{2}+2 \operatorname{Re}\left(M_{s} M_{u}^{*}\right)$ :

$$
\begin{aligned}
& \left|M_{s}\right|^{2}=\frac{1}{2}\left(g \tan \theta_{\mathrm{W}}\right)^{4} \frac{\left(k_{1} p_{1}\right)\left(k_{2} p_{2}\right)}{\left(s-\tilde{m}_{L}^{2}\right)^{2}} ; \\
& \left|M_{u}\right|^{2}=\frac{1}{2}\left(g \tan \theta_{\mathrm{W}}\right)^{4} \frac{\left(p_{1} k_{2}\right)\left(k_{1} p_{2}\right)}{\left(s-\tilde{m}_{L}^{2}\right)^{2}}
\end{aligned}
$$

$$
M_{s} M_{u}^{*}=-\frac{1}{4}\left(g \tan \theta_{\mathrm{W}}\right)^{4} \frac{m_{\chi}^{2}\left(k_{1} k_{2}\right)}{\left(s-\tilde{m}_{L}^{2}\right)\left(u-\tilde{m}_{L}^{2}\right)} .
$$

Cross-section for the $f_{L}+\chi \rightarrow f_{L}+\chi$ scattering at angle $\theta_{12}$ in the system where neutralino is at rest is given by

$$
\left(\frac{d \sigma_{\mathrm{el}}}{d \Omega}\right)_{f_{L \chi}}=\frac{1}{64 \pi^{2} s}|M|^{2}=\frac{\alpha_{\mathrm{e} . \mathrm{m} .}^{2}}{8 \cos ^{4} \theta_{\mathrm{W}}} \frac{\omega^{2}\left(1+\cos \theta_{12}\right)}{\left(m_{\chi}^{2}-\tilde{m}_{L}^{2}\right)^{2}},
$$

where $\omega \gg m_{f}$ is energy of $f_{L}$ in the system where neutralino is at rest, $m_{\chi}$ is the neutralino mass and $\tilde{m}_{L}$ is the mass of the left sfermion.

Let us consider now $f_{R}+\chi \rightarrow f_{R}+\chi$ scattering described by the second term in r.h.s. of Eq. (A1). The diagrams are identical to that in the figure after substituting $f_{L} \rightarrow f_{R}$ and $\tilde{f}_{L} \rightarrow \tilde{f}_{R}$. Since traces do not change when $P_{L} \rightarrow P_{R}$, the expressions (A2)-A4 remain the same, changing only due to coupling constant which increases twice (see Eq. (A1)). Therefore, we obtain

$$
\left(\frac{d \sigma_{\mathrm{el}}}{d \Omega}\right)_{f_{R} \chi}=16\left(\frac{d \sigma_{\mathrm{el}}}{d \Omega}\right)_{f_{L} \chi} .
$$

In this paper we are interested in $\nu+\chi \rightarrow \nu+\chi$ and $e+\chi \rightarrow e+\chi$ scattering. In the former case the crosssection is given by Eq. A5), and in the latter case - by the 
sum of $f_{L}+\chi \rightarrow f_{L}+\chi$ and $f_{R}+\chi \rightarrow f_{R}+\chi$ scattering, i.e. it is by factor 17 larger than the cross-section (A5).

\section{APPENDIX B: KINETIC EQUATION}

In this Appendix we shall study the stage of kinetic equilibrium and the stage after its breaking in the common formalism of kinetic equation similar to [42] and using approach of 44]. We shall confirm in this way the results of Sec. III and clarify the difference in calculations of $M_{\min }$.

Following [44] we introduce the neutralino distribution function $f(x, p, t)$ over comoving coordinates $\vec{x}$ and momenta $\vec{p}=m a^{2} \dot{\vec{x}}$ (with this definition momentum is constant for freely moving particles). The neutralino density is

$$
\rho(x, t)=\frac{m}{a^{3}} \int d^{3} p f(x, p, t)=\bar{\rho}_{\chi}(t)(1+\delta(x, t)) .
$$

The kinetic equation with the collision term of the Fokker-Planck type [46] can be written as

$$
\frac{\partial f}{\partial t}+\frac{p_{i}}{m a^{2}} \frac{\partial f}{\partial x_{i}}-m \frac{\partial \phi}{\partial x_{i}} \frac{\partial f}{\partial p_{i}}=D_{p}(t) \frac{\partial}{\partial p_{i}}\left(\frac{p_{i}}{m T a^{2}} f+\frac{\partial f}{\partial p_{i}}\right),
$$

where $\phi$ is the gravitational potential, which can be neglected at the considered epoch $t \leq t_{\mathrm{e} q}, T(t)$ is the temperature of the ambient plasma given by Eq. (18), and $D_{p}(t)$ is the diffusion coefficient in the momentum space. According to [46]

$$
D_{p}(t)=\frac{40}{3} \int d \Omega \int d \omega n_{0}(\omega)\left(\frac{d \sigma_{e l}}{d \Omega}\right)_{f_{L \chi}}(\delta p)^{2} .
$$

The number 40 in Eq. B33 comes from the counting of degrees of freedom in neutralino-fermion scattering as in the Sec. IIII

The equation (B2) with the diffusion coefficient (B3) coincides with Eq. (16) from [42] except the numerical factor in $D_{p}$ which is of order of unity.

\section{Kinetic decoupling}

Let us consider an exit of neutralinos from the kinetic equilibrium (decoupling) in the homogeneous universe, when $\partial / \partial x_{i}$ terms in Eq. (B2) can be neglected. The temperature of neutralino gas $T_{\chi}$ is defined as

$$
\int p_{i} p_{j} f d^{3} p=\bar{\rho}_{\chi} a^{5} T_{\chi}(t) .
$$

Multiplying Eq. (B2) by $p_{i} p_{j}$ and integrating it over $d^{3} p$ one obtains

$$
\frac{d T_{\chi}}{d t}+2 \frac{\dot{a}}{a} T_{\chi}-\frac{2 D_{p}(t)}{m a^{2}}\left(1-\frac{T_{\chi}(t)}{T(t)}\right)=0,
$$

The initial condition for Eq. (B5) can be chosen at the moment of freezing $t=t_{f}$ as in [42], or more conveniently at any $t_{i}$ from interval $t_{f}<t_{i} \ll t_{d}$, as $T_{\chi}\left(t_{i}\right)=T\left(t_{i}\right)$, where $T$ is the temperature of ambient plasma. Solution of Eq. (B5) (see below) gives transition of ratio $r(t)=T_{\chi}(t) / T(t)$ from $r=1$ to $r_{d}<1$ within some time interval, determined by $r_{d}$. Any value of $t$ in this interval can be taken as definition of decoupling time $t_{d}$. Eq. (B5) and its solution can be simplified using the dimensionless time $\tau=t / t_{d}$. Characteristic time $t_{d}$ is naturally emerged from dimension parameters entering the diffusion coefficient, and up to numerical coefficient it coincides with $t_{d}$ determined in Sec. III] The transition time interval fixes this numerical coefficient with some uncertainty, and we obtain indeed $t_{d}$ (and hence $T_{d}=T\left(t_{d}\right)$ ) approximately equal to those given by Eqs. (19) and (20) in Sec. III The solution of Eq. (B5) in terms of $\tau=t / t_{d}$ is given by

$$
\frac{T_{\chi}(t)}{T_{d}}=\frac{1}{\tau}\left(\tau_{i}^{-1 / 2} e^{1 / 4 \tau^{2}-1 / 4 \tau_{i}^{2}}+\frac{1}{2} e^{1 / 4 \tau^{2}} \int_{\tau_{i}}^{\tau} d^{3} x x^{-5 / 2} e^{1 / 4 x^{2}}\right) .
$$

The asymptotic forms of solution (B6) are given by $T_{\chi} / T_{d}=\tau^{-1 / 2}$ for $\tau \ll 1$ and $T_{\chi} / T_{d}=\tau^{-1} \Gamma(3 / 4) / 2^{1 / 2}$ for $\tau \gg 1$ as it must be. From solution $(\mathbb{B} 6)$ it is seen that transition from kinetic equilibrium of neutralino with relativistic fermions to the non-equilibrium regime proceeds very fast. By this reason our consideration of diffusion and free streaming independently in Sec. IIII is well justified.

\section{Diffusion}

Consider Eq. (B2) before kinetic decoupling, $t \ll t_{d}$. One can find the first two moments by integrating first time Eq. (B2) over $d^{3} p$ and second time over $p_{i} d^{3} p$. Inserting the first of the obtained equation into the second one we obtain the following equation for the Fourier components:

$$
\frac{\partial^{2} \delta}{\partial^{2} t}+2 \frac{\dot{a}}{a} \frac{\partial \delta}{\partial t}+D_{p}(t) \frac{1}{m T a^{2}} \frac{\partial \delta}{\partial t}=\frac{k_{i} k_{j}}{\bar{\rho}_{\chi} a^{7} m} \int p_{i} p_{j} f d^{3} p .
$$

The r.h.s. of the (B7) has a tensor form

$$
\frac{1}{\bar{\rho}_{\chi} a^{7} m} \int p_{i} p_{j} f d^{3} p=E \delta_{i j}+F k_{i} k_{j},
$$

where the isotropic part $E=T_{\chi} \delta_{k} / a^{2} m$ for any $\tau$, while $F$ depends on time $t$. In the limit $\tau \ll 1$ we may put $F=0$ and neglect the first and second terms in (B7). The resultant equation coincides with diffusion equation (24) with the same diffusion coefficient (23) and has the same solution.

In 42] only this diffusion limit of the general kinetic equation (B2) has been considered. 


\section{Free streaming}

In the limiting case $\tau \gg 1$, i.e. after decoupling, the (B2) has a simple form

$$
\frac{\partial f}{\partial t}+\frac{p_{i}}{m a^{2}} \frac{\partial f}{\partial x_{i}}=0
$$

with the solution

$$
f \propto \exp \left[\frac{i k_{j} p_{j}}{m a_{d}} g(t)\right],
$$

where $g(t)$ is the same function as (28). The solution (B10) is valid with a good accuracy also at $\tau \geq 1$, because according to (B6), kinetic decoupling proceeds very fast. Integrating (B10) over $d^{3} p$ with initial condition

$$
f\left(t_{d}\right)=\left(2 \pi T_{d} m a_{d}^{2}\right)^{-3 / 2} \exp \left\{-\frac{p^{2}}{2 T_{d} m a_{d}^{2}}\right\}
$$

one obtains

$$
n_{\vec{k}}(t)=n_{\vec{k}}\left(t_{d}\right) e^{-(1 / 2) k^{2} g^{2}(t) T_{d} / m_{\chi}},
$$

and then Eqs. (33), (34) and (38) from Sec. III
[1] J. Silk and A. Stebbins, Astrophys. J. 411, 439 (1993).

[2] A. V. Gurevich, K. P. Zybin, and V. A. Sirota, Sov. Phys. Usp. 167, 913 (1997).

[3] B. Moore et al., Astrophys. J. Lett. 524, L19 (1999).

[4] S. Ghigna, B. Moore, F. Governato, G. Lake, T. Quinn, and J. Stadel, Astrophys. J. 544, 616 (2000).

[5] A. Klypin, S. Gottlober, A. V. Kravtsov, and A. M. Khokhlov, Astrophys. J. 516, 530 (2002).

[6] E. Bertschinger, Astrophys. J. Suppl. 58, 39 (1985).

[7] Y. Hoffman and J. Shaham, Astrophys. J. 297, 16 (1985).

[8] B. S. Ryden and J. E. Gunn, Astrophys. J. 318, 39 (1987).

[9] A. V. Gurevich and K. P. Zybin, Sov. Phys. Usp. 165, 723 (1995).

[10] J. F. Navarro, C. S. Frenk, and S. D. M. White, Astrophys. J. 462, 563 (1996).

[11] D. D. Kelson et al., Astrophys. J. 576, 720 (2002).

[12] Y. P. Jing and Y. Suto, Astrophys. J. Lett. 529, 69 (2000).

[13] D. Syer and S. D. M. White, Mon. Not. R. Astron. Soc. 293, 337 (1998).

[14] A. Dekel, I. Arad, J. Devor, and Y. Birnboim, astro-ph/0205448

[15] J. E. Taylor and J. F. Navarro, Astrophys. J. 563, 483 (2001).

[16] V. S. Berezinsky, A. V. Gurevich, and K. P. Zybin, Phys. Lett. B 294, 221 (1992).

[17] V. Berezinsky, A. Bottino, and G. Mignola, Phys. Lett. B 325, 136, (1994).

[18] L. Bergstrom, J. Edsjo, P. Gondolo, and P. Ulio, Phys. Rev. D 59, 043506 (1999).

[19] P. Gondolo and J. Silk, Phys. Rev. Lett. 83, 1719 (1999).

[20] P. Ullio, H. Zhao, and M. Kamionkowski, Phys. Rev. D 64, 043504 (2001).

[21] G. Bertone, G. Sigl, and J. Silk, Mon. Not. R. Astron. Soc. 337, 98 (2002).

[22] D. Merritt, M. Milosavljevic, L. Verde, and R. Jimenez, Phys. Rev. Lett. 88, 191301 (2002).

[23] V. Berezinsky, A. Bottino, and G. Mignola, Phys. Lett. B 391355 (1997).

[24] G. Jungman and M. Kaminkowski Phys. Rev. D 49, 2316 (1994).

[25] A. Bottino, C. Favero, N. Fornengo, and G. Mignola Astropat. Phys., 3, 77 (1995).
[26] A. Bottino, F. Donato, N. Fornengo, and P. Salati, Phys. Rev. D 58, 123503 (1998).

[27] R. Aloisio, P. Blasi, and A. V. Olinto, astro-ph/0206036

[28] A. Tasitsiomi and A. V. Olinto, Phys. Rev. D 66, 083006 (2002).

[29] J. E. Taylor and J. Silk, submitted to Mon. Not. R. Astron. Soc., astro-ph/0207299

[30] L. Bergstrom, J. Edsjo, and P. Ulio, Phys. Rev. D 58, 083507 (1998).

[31] L. Bergstrom, J. Edsjo, and C. Gunnarsson, Phys. Rev. D 63, 083515 (2001).

[32] P. Ullio, L. Bergstrom, J. Edsjo, and C. Lacey, Phys. Rev. D 66, 123502 (2002).

[33] P. Blasi, A. V. Olinto, and C. Tyler, astro-ph/0202049

[34] B. L. Carr and C. C. Lacey, Astrophys. J., 316, 23 (1987).

[35] E. Ardi, T. Tsuchiya, and A. Burkert, astro-ph/0206026

[36] E. W. Kolb and I. I. Tkachev, Phys. Rev. D 50, 769 (1994).

[37] V. I. Dokuchaev and Yu. N. Eroshenko, JETP 94, 5 (2002).

[38] W. H. Press and P. Schechter, Astrophys. J. 187, 425 (1974).

[39] J. R. Bond, S. Cole, G. Efstathiou, and N. Kaiser, Astrophys. J. 379, 440 (1991).

[40] C. Lacey and S. Cole, Mon. Not. R. Astron. Soc. 262, 627 (1993).

[41] J. E. Gunn et al., Astrophys. J. 223, 1015 (1978).

[42] K. P. Zybin, M. I. Vysotsky, and A. V. Gurevich, Phys. Lett. A 260, 262 (1999).

[43] D. J. Schwarz, S. Hofmann, and H. Stocker, Phys. Rev. D 64, 083507 (2001).

[44] P. J. E. Peebles, Large-Scale Structure of the Universe (Princeton University Press, Princeton, USA, 1993)

[45] B. Moore et al., Astrophys. J. Lett., 524, 19 (1999).

[46] L. D. Landau, E. M. Lifshits, and L. P. Pitaevsky, Course of Theoretical Physics: Physical kinetics, vol. 10, (Pergamon Press, 1981).

[47] J. M. Bardeen et al., Astrophys. J. 304, 15 (1986).

[48] A. Burkert, Astrophys. J. Lett., 447, 25 (1995).

[49] W. J. G. de Blok, A. Bosma, and S.S. McGaugh, astro-ph/0212102

[50] O. Y. Gnedin, L. Hernquist, and J. P. Ostriker, Astrophys. J. 514, 109 (1999).

[51] D. N. Spergel et al., Astrophys. J., submitted, 
astro-ph/0302209

[52] E. V. Bugaev and K. V. Konishchev, Phys. Rev. D 65, 123005 (2002).

[53] C. Schmid, D. J. Schwarz, and P. Widerin, Phys. Rev. D 59, 043517 (1999).

[54] V. S. Berezinsky and A. A. Mikhailov, Phys. Lett. B 449, 237 (1999).

[55] C. G. Lacey and J. P. Ostriker, Astrophys. J. 299, 633 (1985).
[56] S. Coutu et al., (HEAT-pbar Collaboration), in Proceedings of 27th ICRC (2001).

[57] E. A. Baltz, J. Edsjoet, K. Freese, and P. Gondolo, astro-ph/0211239

[58] G. Jungman, M. Kamionkowski, and K. Griest, Phys. Rep. 267, 195 (1996).

[59] H. E. Haber and G. L. Kane, Phys. Rep. 117, 75 (1985). 